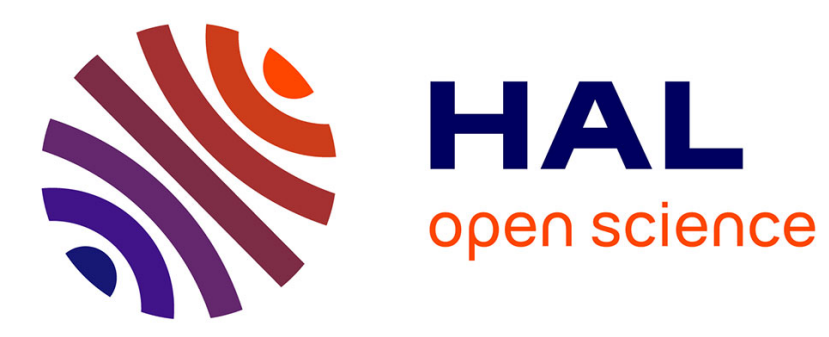

\title{
Analysis of a Tripartite Entanglement Distribution Switch
}

Philippe Nain, Gayane Vardoyan, Saikat Guha, Don Towsley

\section{To cite this version:}

Philippe Nain, Gayane Vardoyan, Saikat Guha, Don Towsley. Analysis of a Tripartite Entanglement Distribution Switch. 2021. hal-03195985v2

\section{HAL Id: hal-03195985 \\ https://hal.inria.fr/hal-03195985v2}

Preprint submitted on 14 Apr 2021 (v2), last revised 16 Jan 2022 (v5)

HAL is a multi-disciplinary open access archive for the deposit and dissemination of scientific research documents, whether they are published or not. The documents may come from teaching and research institutions in France or abroad, or from public or private research centers.
L'archive ouverte pluridisciplinaire HAL, est destinée au dépôt et à la diffusion de documents scientifiques de niveau recherche, publiés ou non, émanant des établissements d'enseignement et de recherche français ou étrangers, des laboratoires publics ou privés. 


\title{
Analysis of a Tripartite Entanglement Distribution Switch
}

\author{
Philippe Nain ${ }^{1}$, Gayane Vardoyan ${ }^{2}$, Saikat Guha ${ }^{3}$, and Don Towsley ${ }^{4}$ \\ ${ }^{1}$ Inria, France; philippe.nain@inria.fr \\ ${ }^{2}$ QuTech, TU Delft, The Netherlands; gvardoyan@tudelft.nl \\ ${ }^{3}$ The University of Arizona, Tucson, AZ, USA; saikat@optics.arizona.edu \\ ${ }^{4}$ University of Massachusetts Amherst, MA 01003, USA; towsley@cs.umass.edu
}

April 14, 2021

\begin{abstract}
We study a quantum switch that distributes tripartite entangled states to sets of users. The entanglement switching process requires two steps: first, each user attempts to generate bipartite entanglement between itself and the switch; and second, the switch performs local operations and a measurement to create multipartite entanglement for a set of three users. In this work, we study a simple variant of this system, wherein the switch has infinite memory and the links that connect the users to the switch are identical. This problem formulation is of interest to several distributed quantum applications, while the technical aspects of this work result in new contributions within queueing theory. The state of the system is modeled as continuous time Markov chain (CTMC) and performance metrics of interest (probability of an empty system, switch capacity, expectation and variance of the number of qubit-pairs stored) are computed via the solution of a two-dimensional functional equation obtained by reducing it to a boundary value problem on a closed curve. This work is a follow up of [28] where a switch distributing entangled multipartite states to sets of users was studied but only the switch capacity and the expected number of stored qubits were derived.
\end{abstract}

Keywords: Quantum switch; Markov process; Queueing; Boundary value problem.

\section{Introduction}

Entanglement is an essential component of quantum computation, information, and communication. Its applications include quantum cryptography (e.g., $[3,4,12])$, distributed quantum computing (e.g., [9, 22]), and quantum sensing (e.g., multipartite entanglement for quantum metrology [18] and spectroscopy [24]). These applications drive the increasing need for a quantum switching network that can supply end-to-end entanglement to groups of endpoints that request them [30, 33, 35]. To realize such quantum systems, several architectures have been proposed to support high entanglement generation rates, high fidelity, and long coherence times $[1,5,19,20,23,25]$. In this paper, we focus on the most basic and fundamental 
component of a quantum network - a single quantum switch that serves $k$ users in a star topology. Each user has a dedicated link connected to the switch as shown in Figure 1 (a detailed description of this figure is provided below). In the most general case, the switch serves $n$-partite entangled states to sets of users according to incoming requests, where $n \leq k$. To achieve this, link-level entangled states are generated at a constant rate across each link, resulting in two-qubit maximally-entangled states (i.e., Bell pairs or EPR states). These qubits are stored at local quantum memories: one from each Bell pair at the user and the other one at the switch. We consider the algorithm where the switch performs multi-qubit measurements to provide end-to-end entanglement to user groups of size $n$ when enough of these bipartite states are accrued (at least $n$ of them). When $n=2$, the switch uses Bell-state measurements (BSMs) and when $n \geq 3$, it uses $n$-qubit Greenberger-Horne-Zeilinger (GHZ) basis measurements [29]. For additional background on quantum switches the interested reader is referred to [36, Section 2] and [37, Section II].

Figure 1 depicts a quantum switch serving $k$ users in a star topology. Each user is connected to the switch via a dedicated connection or link (e.g., optical fiber). All nodes (including the switch) are assumed to have quantum memories, or buffers. When bipartite entanglement is successfully generated on a link, a user's qubit becomes entangled with a qubit located at the switch (represented with black dashed line in the figure). When entanglement is generated between the switch and a second user, the switch may perform a Bell state measurement (BSM) on its two locally-held entangled qubits; as a result, the two user's qubits become entangled up to local Pauli corrections. This process can be generalized to three or more users, with the modification of a GHZ-basis measurement instead of a BSM at the switch. The result is a GHZ state entangling the users. In this paper we consider the situation when three user's qubits are entangled (i.e., $n=3$ ), links are homogeneous, and all buffers are infinite. The situation when $n=2$ was studied in [37] under a variety of assumptions.

Let us now introduce the problem in abstract, mathematical terms. As we shall see, while the problem considered in this work was initially motivated by its application to entanglement switching, its relevance reaches far beyond this example, and it is of interest to queueing theory in general. Consider a server and $k$ clients in a star topology like in Figure 1. Each client has a dedicated link to the server; one may think of these links either as communication channels or physical paths that may be used for object delivery. For instance, in the former scenario the clients may send data packets to the server for processing, and in the latter, the central node may be an assembly plant that receives components of a product from geographically distant manufacturing plants. (As soon as $n$ components (or parts) from $n$ distinct clients arrive at the central node, they are processed (or assembled) and immediately leave the system.) We assume that the processing/assembly step is instantaneous, but succeeds with probability $q$ (the fact that the assembly step can fail is important in the context of a quantum switch). Finally, assume that the arrival process on link $l(l=1, \ldots, k)$ is a Poisson process with a constant rate $\mu>0$ and that these $k$ arrival processes are mutually independent. Each link is equipped with an infinite buffer and so is the switch.

If we denote by $Q_{l}(t)$ the number of entangled qubit-pairs stored by link $l$ at time $t$, the process $\left\{\left(Q_{1}(t), \ldots, Q_{k}(t)\right)\right\}_{t}$ is a continuous-time Markov chain (CTMC). As a consequence of the algorithm described above, when $n=2$, at most one component of the vector $\left(Q_{1}(t), \ldots, Q_{k}(t)\right)$ is non-zero. Exploiting this property, the state of the switch can be represented by a birth and death process thereby yielding the stationary distribution - and from it the main performance measures of interest - explicitly 
or in closed-form both when the switch has a finite or infinite memory [37]. When the memory is infinite it is shown in [37] that the system is stable when $k>2$ and, among other results, that the system capacity - defined as the maximum achievable number of successful assemblies per time unit - is $q \sum_{l=1}^{k} \mu_{l} / 2$ when the switch has an infinite memory. Results are also obtained in [37] when quantum state decoherence and an associated cut-off policy are added to the model, which amounts to assuming that stored entanglements have an exponential lifetime with a constant rate.

Getting explicit/closed-form results for $n \geq 3$ when link rates are arbitrary is very challenging. Even when all links are identical, say $\mu:=\mu_{l}$ for $l=1, \ldots, k$, and the switch has infinite memory, the problem becomes significantly more difficult to analyze when $n \geq 3$. In particular, the stability analysis requires the introduction of a Markov chain embedded in the original Markov chain. Via a careful choice of a Lyapunov function and application of Foster's theorem, the ergodicity condition of the embedded Markov chain, yielding the ergodicity condition of the original one, is found to be $k>n$ in [28]. In [28] the switch capacity and the expected number of stored qubits at the switch are also derived and are given by $\frac{q \mu k}{n}$ and $\frac{k(n-1)}{2(k-n)}$, respectively. The latter results are obtained by setting the drift of appropriate Lyapounov functions to zero. These are all first-order moment properties. The objective of this work is to get higherorder moment properties and, in particular, the variance of the number of stored qubits when $n=3$. In the setting of entanglement switching, the case $n=3$ is referred to as tripartite entanglement distribution. With a slight abuse of notation, the number of stored qubits will refer to the number of qubits located in the switch memory.

As noted above this model extends far beyond entanglement switching. In general, one may view the system as a stochastic assembly-like queue, or a "kitting" process, e.g., as in [21, 31, 34], since in a sense, the switch "assembles" multipartite entangled states from bipartite ones, whose "arrival" into the system is driven by a stochastic process. Interestingly, none of these similar problem formulations found in literature have a direct correspondence to our problem, as in our case the number of links or users being serviced by the central node is allowed to be, in theory, infinite, and our goal is to derive exact results, as opposed to approximate ones, or bounds. Hence, the problem studied here is novel, and the results derived in this work are of independent interest to queueing theory.

The paper is organized as follows: in Section 2 we identify a two-dimensional CTMC that models the state of the switch when $n=3$ and write a two-dimensional functional equation satisfied by its generating function. The main performance metrics are expressed in Section 3 either in explicit form (we retrieve the capacity and expected number of stored entangled qubits found in [28] and reported above) or in terms of the generating function (variance). In Section 4 we solve the functional equation when the number of users is infinite (i.e., $k=\infty$ ) and derive the variance of the number of stored qubits. For finite $k$ the functional equation is solved in Section 5 by reducing it to the solution of a boundary value problem (BVP) on a closed curve. From it, we derive in Section 6 the variance of the number of stored qubits. Formulas obtained for the probability that the system is empty and for the variance of the number of stored qubits are amenable to numerical evaluation as shown in Section 7 . The paper is concluded in Section 8 .

A word on the notation. Let $\mathbb{N}=\{0,1,2, \ldots\}$ denote the set of nonnegative integers and $\underline{e}_{l}$ the unit 
vector in $\mathbb{N}^{k}$ whose entries all equal zero except for the $l$ th one that equals one. The first, second, and third derivatives at point $x$, when they exist, of a function $\varphi$ are denoted by $\varphi^{\prime}(x), \varphi^{\prime \prime}(x)$, and $\varphi^{\prime \prime \prime}(x)$, respectively.

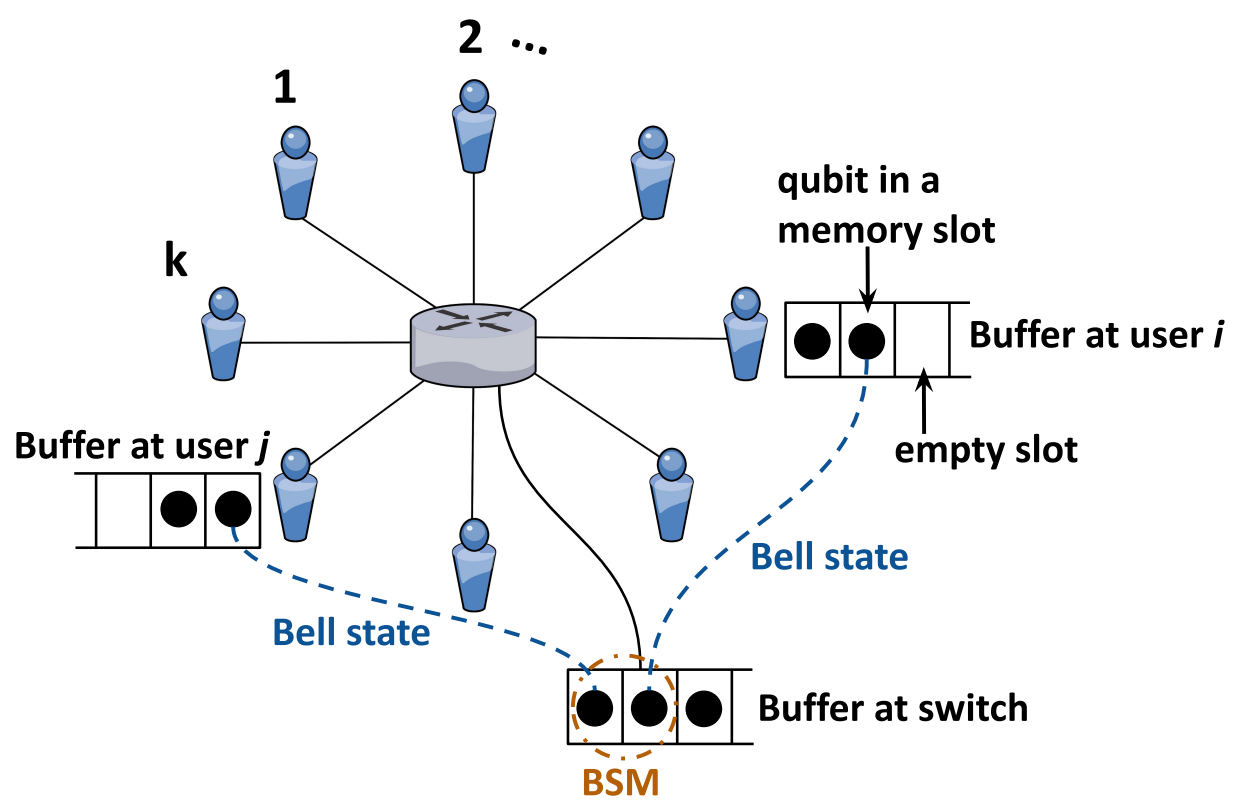

Figure 1: A quantum switch serving $k$ users in a star topology.

\section{The model}

Throughout the paper we assume that the stability condition $k>3$ holds [28].

Let $Q_{l}(t)$ denote the number of entangled qubits stored by link $l=1, \ldots, k$. At time $t$, the state of the switch can be represented by the vector $\left(Q_{1}(t), \ldots, Q_{n}(t)\right)$, whose state-space is $\mathcal{S}=\left\{i \underline{e}_{l}+j \underline{e}_{l^{\prime}}\right.$ : $\left.i, j \in \mathbb{N}, l, l^{\prime} \in\{1,2, \ldots, k\}, l \neq l^{\prime}\right\}$ since at most two links can store qubit-pairs at the same time. For mathematical convenience we will instead use a more compact representation of the state of the switch, which we now describe.

The state of the switch will be represented by the two-dimensional irreducible continuous-time Markov chain (CTMC) $\{(X(t), Y(t)), t \geq 0\}$ on $\mathbb{N}^{2}$, whose non-zero transitions are displayed in Figure 2 . In this representation, states $(i, j)$ and $(j, i)$, for $i, j \in \mathbb{N}$ are equivalent. We choose to differentiate between them because it simplifies the analysis of the model, despite increasing the number of total states in the CTMC. Consider state $(0,0)$ when no qubit-pairs are stored at the links - referred to as the empty system. From 
there, an entanglement is generated on one of the $k$ links at rate $k \mu$; hence, we split this rate in half among the states $(0,1)$ and $(1,0)$. The state $(i, 0)$ (resp. $(0, i))$ corresponds to the situation where one link stores $i$ qubit-pairs and the $k-1$ other links do not store anything. From there, the system may evolve to state $(i+1,0)$ at rate $\mu$ if an additional entanglement is generated on the busy link, and to state $(i, 1)$ at rate $(k-1) \mu$ if an entanglement is generated on of the $k-1$ non-busy links. Finally, state $(i, j) \in\{1,2, \ldots\}^{2}$ (resp. $\left.(j, i) \in\{1,2, \ldots\}^{2}\right)$ describes the situation where two links are storing qubit-pairs, one is storing $i$ qubit-pairs and the other one is storing $j$ qubit-pairs. From this state, the system may join state $(0,0)$ if an entanglement appears on one of the $k-2$ empty links, which occurs at rate $(k-2) \mu$, or join state $(i+1, j)(\operatorname{resp}(i, j+1))$ if an additional entanglement is generated, at rate $\mu$, at one of the two busy links.

For convenience we temporarily introduce the rates $\lambda=\mu$ and $\nu=(k-2) \mu$. These rates can be interpreted as arrival and departure rates, respectively. The non-zero transition rates of the CTMC $\{(X(t), Y(t)), t \geq 0\}$ (see Figure 2) are

$$
\begin{array}{cl} 
& (0,0) \rightarrow(1,0) \quad \text { with rate } \frac{2 \lambda+\nu}{2}, \\
& (0,0) \rightarrow(0,1) \quad \text { with rate } \frac{2 \lambda+\nu}{2}, \\
\text { For } i \geq 1: \quad(i, 0) \rightarrow(i, 1) \quad \text { with rate } \lambda+\nu, \\
\text { For } j \geq 1: \quad(0, j) \rightarrow(1, j) \quad \text { with rate } \lambda+\nu, \\
\text { For } i \geq 1: \quad(i, 0) \rightarrow(i+1,0) \quad \text { with rate } \lambda, \\
\text { For } j \geq 1: \quad(0, j) \rightarrow(0, j+1) \quad \text { with rate } \lambda, \\
\text { For } i \geq 1, j \geq 1: \quad(i, j) \rightarrow(i, j+1) \quad \text { with rate } \lambda, \\
\text { For } i \geq 1, j \geq 1: \quad(i, j) \rightarrow(i+1, j) \quad \text { with rate } \lambda, \\
\text { For } i \geq 1, j \geq 1: & (i, j) \rightarrow(i-1, j-1) \quad \text { with rate } \nu .
\end{array}
$$

Let $\pi(i, j)$ be the steady-state probability that the chain is in state $(i, j)$. The balance equations read

$$
\begin{aligned}
& (2 \lambda+\nu) \pi(0,0)=\nu \pi(1,1), \\
& (2 \lambda+\nu) \pi(1,0)=\frac{(2 \lambda+\nu)}{2} \pi(0,0)+\nu \pi(2,1), \\
& (2 \lambda+\nu) \pi(0,1)=\frac{(2 \lambda+\nu)}{2} \pi(0,0)+\nu \pi(1,2), \\
& (2 \lambda+\nu) \pi(1,1)=\nu \pi(2,2)+(\lambda+\nu) \pi(1,0)+(\lambda+\nu) \pi(0,1), \\
& (2 \lambda+\nu) \pi(i, 0)=\nu \pi(i+1,1)+\lambda \pi(i-1,0), \quad i \geq 2, \\
& (2 \lambda+\nu) \pi(0, j)=\nu \pi(1, j+1)+\lambda \pi(0, j-1), \quad j \geq 2, \\
& (2 \lambda+\nu) \pi(i, 1)=\nu \pi(i+1,2)+\lambda \pi(i-1,1)+(\lambda+\nu) \pi(i, 0), \quad i \geq 2, \\
& (2 \lambda+\nu) \pi(1, j)=\nu \pi(2, j+1)+(\lambda+\nu) \pi(0, j)+\lambda \pi(1, j-1), \quad j \geq 2, \\
& (2 \lambda+\nu) \pi(i, j)=\nu \pi(i+1, j+1)+\lambda \pi(i-1, j)+\lambda \pi(i, j-1), \quad i \geq 2, j \geq 2 .
\end{aligned}
$$






Figure 2: CTMC for a system with $k$ homogeneous links and a switch that has infinite buffer and serves only tripartite entanglement. $\mu$ is the entanglement generation rate at the link level.

Define the generating function

$$
F(x, y)=\sum_{i, j \geq 0} \pi(i, j) x^{i} y^{j}, \quad|x| \leq 1,|y| \leq 1 .
$$

It is shown in Appendix A that for $|x| \leq 1,|y| \leq 1$,

$$
\begin{aligned}
\left(\lambda(1-x)+\lambda(1-y)+\nu\left(1-\frac{1}{x y}\right)\right) F(x, y) & =\nu\left(y-\frac{1}{x y}\right) F(x, 0)+\nu\left(x-\frac{1}{x y}\right) F(0, y) \\
& -\nu\left(\frac{x+y}{2}-\frac{1}{x y}\right) F(0,0) .
\end{aligned}
$$

Replacing $\lambda$ by $\mu$ and $\nu$ by $(k-2) \mu$ in (10), and multiplying by $-x y / \mu$ both sides of the resulting equation, yields

$$
K(x, y) F(x, y)=(k-2)[a(x, y) F(x, 0)+b(x, y) F(0, y)+c(x, y) F(0,0)], \quad|x| \leq 1,|y| \leq 1,
$$

with

$$
\begin{aligned}
K(x, y) & :=x^{2} y+x y^{2}-k x y+k-2, \\
a(x, y) & :=1-x y^{2} \\
b(x, y) & :=1-x^{2} y \\
c(x, y) & :=\frac{x^{2} y+x y^{2}}{2}-1 .
\end{aligned}
$$


Notice that (11) exhibits the following symmetry

$$
F(x, y)=F(y, x)
$$

for all $|x| \leq 1,|y| \leq 1$. When $K(x, x) \neq 0$ and $x=y$ it follows from (11) that

$$
F(x, x)=(k-2)\left(\frac{a(x, x)+b(x, x)}{K(x, x)} F(x, 0)+\frac{c(x, x)}{K(x, x)} F(0,0)\right),
$$

since $F(x, 0)=F(0, x)$. Letting $x \rightarrow 1$ in $(14)$, we get by noting that $F(1,1)=1$ and by using L'Hôpital's rule in the r.h.s. that

$$
1=(k-2)\left(\frac{3}{k-3} F(1,0)-\frac{3}{2(k-3)} F(0,0)\right),
$$

yielding

$$
2 F(1,0)-F(0,0)=\frac{2(k-3)}{3(k-2)}
$$

\section{Main performance metrics}

Let $N$ denote the total number of stored entangled qubits at the switch in steady state. Via a Lyapounov function approach, the expectation of $N$ has been computed in [28] in the general case where any combination of $n$ users out of the $k$ users want to communicate, and was found to be $\mathbb{E}[N]=\frac{k(n-1)}{2(k-n)}$. When $n=3$ as in the present paper, then $\mathbb{E}[N]=\frac{k}{k-3}$. We also obtained in [28] that the capacity $C$ of the switch, defined as the maximum achievable number of successful assemblies (or entanglement swappings) per time unit, is given by $C=\mu q \frac{k}{n}$, where $q$ is the probability that an entanglement swapping succeeds.

For the sake of completeness we show in Sections 3.1 and 3.2 how, when $n=3, C$ and $\mathbb{E}[N]$ can be retrieved from the functional equation (11). Interestingly enough these calculations can be performed without solving this equation. This is in contrast with the variance, which cannot be obtained without solving (11), as discussed in Section 3.3.

\subsection{Capacity}

A GHZ measurement can be generated from any state $(i, j)$ for $i, j \geq 1$ : this occurs with rate $\nu=(k-2) \mu$, whenever a third link (not one of the two links that has a stored entanglement) generates an entanglement. Hence, the system capacity $C$ is

$$
C=q \nu \sum_{\substack{i \geq 1 \\ j \geq 1}} \pi(i, j)=q \nu(1-F(1,0)-F(0,1)+F(0,0))=q \mu(k-2)(1-2 F(1,0)+F(0,0))=\mu q \frac{k}{3},
$$

by $F(1,0)=F(0,1)$ from (13) and (15). 


\subsection{Expectation}

Since $\mathbb{E}[N]=\left.\frac{d}{d x} F(x, x)\right|_{x=1}$, let $y=x$ in (11), differentiate both sides of the resulting equation with respect to $x$ and then set $x=1$. This gives by using (15)

$$
\mathbb{E}[N]=\frac{k+\left.6(k-2) \frac{d}{d x} F(x, 0)\right|_{x=1}}{2(k-3)} .
$$

Let us calculate $\left.\frac{d}{d x} F(x, 0)\right|_{x=1}$.

It is shown in Lemma 2 in Appendix B that for fixed $|x|=1, K(x, y)$ defined in (12) has a unique zero $y=y(x)$ such that $|y(x)| \leq 1$.

Since $F(x, y)$ is well-defined for $|x| \leq 1,|y| \leq 1$, the l.h.s. of (11) must vanish when $(x, y)=(x, y(x))$ with $|x|=1$. This gives for $|x|=1$

$$
0=\left(x y(x)^{2}-1\right) F(x, 0)+\left(x^{2} y(x)-1\right) F(0, y(x))-\left(\frac{x^{2} y(x)+x y^{2}(x)}{2}-1\right) F(0,0) .
$$

By twice differentiating (18) with respect to $x$, we obtain for $|x|=1$

$$
\begin{aligned}
0= & {\left[4 y(x) y^{\prime}(x)+2 x y^{\prime}(x)^{2}+2 x y(x) y^{\prime \prime}(x)\right] F(x, 0)+2\left[y^{2}(x)+2 x y(x) y(x)\right] \frac{d}{d x} F(x, 0) } \\
& +\left[2 y(x)+4 x y^{\prime}(x)+x^{2} y^{\prime \prime}(x)\right] F(0, y(x))+2\left[2 x y(x)+x^{2} y^{\prime}(x)\right] y^{\prime}(x) \frac{d}{d y} F(0, y(x)) \\
& +\left[x^{2} y(x)-1\right] y^{\prime \prime}(x) F_{y}^{\prime}(0, y(x))+\left[x^{2} y(x)-1\right] y^{\prime}(x)^{2} \frac{d^{2}}{d y^{2}} F(0, y(x)) \\
& -\left[2 y(x)+4 x y^{\prime}(x)+4 y(x) y^{\prime}(x)+x^{2} y^{\prime \prime}(x)+2 x y^{\prime}(x)^{2}+2 x y(x) y^{\prime \prime}(x)\right] \frac{F(0,0)}{2} .
\end{aligned}
$$

By setting $x=1$ in the above equation and using $y(1)=1, y^{\prime}(1)=-1$ and $y^{\prime \prime}(1)=\frac{2(k-2)}{k-3}$ (see Lemma 2 ), we readily find that (Hint: $\left.\frac{d}{d x} F(x, 0)\right|_{x=1}=\left.\frac{d}{d y} F(0, y)\right|_{y=1}$ because $F(x, y)=F(y, x)$ for all $|x| \leq 1$, $|y| \leq 1$ as observed in Section 2)

$$
\left.\frac{d}{d x} F(x, 0)\right|_{x=1}=\frac{\left(-4+3 y^{\prime \prime}(1)\right)(2 F(1,0)-F(0,0)}{8}=\frac{k}{6(k-2)},
$$

by using (15). Hence, by (17),

$$
E[N]=\frac{k}{k-3}
$$




\subsection{Variance}

To obtain the variance of $N$, let us twice differentiate both sides of (11) with respect to $x$ after setting $y=x$ and then set $x=1$. We show in Appendix $\mathrm{C}$ that

$$
\begin{aligned}
\left.\frac{d^{2}}{d x^{2}} F(x, x)\right|_{x=1} & =\frac{1}{k-3}\left(\frac{2 k}{3}-(k-6) \mathbb{E}[N]+\left.6(k-2) \frac{d}{d x} F(x, 0)\right|_{x=1}+\left.3(k-2) \frac{d^{2}}{d x^{2}} F(x, 0)\right|_{x=1}\right), \\
& =\frac{k(2 k+3)}{3(k-3)^{2}}+\left.\frac{3(k-2)}{k-3} \frac{d^{2}}{d x^{2}} F(x, 0)\right|_{x=1}
\end{aligned}
$$

by using (20) and (21). The variance of $N$ is then obtained via the formula

$$
\begin{aligned}
\operatorname{var}(N) & =\left.\frac{d^{2}}{d x^{2}} F(x, x)\right|_{x=1}+\mathbb{E}[N]-(\mathbb{E}[N])^{2} \\
& =\frac{2 k}{3(k-3)}+\left.\frac{3(k-2)}{k-3} \frac{d^{2}}{d x^{2}} F(x, 0)\right|_{x=1},
\end{aligned}
$$

by using (21) and (22).

It remains to find $\left.\frac{d^{2}}{d x^{2}} F(x, 0)\right|_{x=1}$ in $(23)$. Unfortunately this quantity cannot be obtained without solving equation (11). Indeed, the interested reader can check that differentiating (19) w.r.t. $x$ yields the tautology ' $0=0$ '; differentiating (19) twice w.r.t. $x$ gives an equation involving the third and fourth derivative of $F(x, 0)$ at $x=1$. Continuing to differentiate (19) generates an open system of equations (i.e., there are more unknowns than the number of equations) from which we cannot derive $\left.\frac{d^{2}}{d x^{2}} F(x, 0)\right|_{x=1}$.

The rest of the paper is devoted to the solution of (11), which will allow us to compute $\left.\frac{d^{2}}{d x^{2}} F(x, 0)\right|_{x=1}$ and subsequently $\operatorname{var}(N)$.

\section{Infinite number of users}

Dividing both sides of (11) and letting $k \rightarrow \infty$ yields

$$
(1-x y) F(x, y)=\left(1-x y^{2}\right) F(x, 0)+\left(1-x^{2} y\right) F(0, y)+\frac{x^{2} y+x y^{2}-2}{2} F(0,0), \quad|x| \leq 1,|y| \leq 1 .
$$

Setting $y=1 / x$ for $|x|=1$ (or equivalently $y=\bar{x}$ ) and noting that $|y|=1$ yields (Hint: we use that $F(x, 1 / x)$ is well-defined for $|x|=1$ so that the l.h.s. of (24) must vanish when $y=1 / x)$

$$
0=\left(1-\frac{1}{x}\right) F(x, 0)+(1-x) F(0,1 / x)+\frac{x+1 / x-2}{2} F(0,0), \quad|x|=1,
$$

which can be rewritten as

$$
F(x, 0)=x F(0,1 / x)+\frac{1-x}{2} F(0,0), \quad|x|=1 .
$$


The function $F(x, 0)$ is analytic for $|x|<1$ and continuous for $|x| \leq 1$ and the function $F^{-}(x):=$ $x F(0,1 / x)+\frac{1-x}{2} F(0,0)$ is analytic in $|x|>1$ and continuous in $|x| \geq 1$ with a pole at $x=\infty$. Since $F(x, 0)$ and $F^{-}(x)$ are each other's analytic continuations from (25), Liouville's theorem implies that $F(x, 0)=P(x)$ and $x F(0,1 / x)+\frac{1-x}{2} F(0,0)=P(x)$ for all $x \in \mathbb{C}$. where $P(x)$ is an arbitrary polynomial of degree one. With $P(x)=a_{0}+a_{1} x$ we obtain, for $x \in \mathbb{C}$,

$$
\begin{aligned}
F(x, 0) & =a_{0}+a_{1} x, \\
x F(0,1 / x)+\frac{1-x}{2} F(0,0) & =a_{0}+a_{1} x .
\end{aligned}
$$

Letting $x=0$ in (26) implies that $a_{0}=F(0,0)$. On the other hand, letting $x=1$ in (26) implies that $a_{1}=F(1,0)-F(0,0)$. By using (15) we get that $a_{1}=\frac{1}{3}-\frac{1}{2} F(0,0)$. Hence, for $x \in \mathbb{C}$,

$$
\begin{aligned}
F(x, 0) & =F(0,0)+\left(\frac{1}{3}-\frac{1}{2} F(0,0)\right) x, \\
x F(0,1 / x)+\frac{1-x}{2} F(0,0) & =F(0,0)+\left(\frac{1}{3}-\frac{1}{2} F(0,0)\right) x .
\end{aligned}
$$

It remains to identify $F(0,0)$. Dividing (29) by $x$ and letting $x \rightarrow \infty$ yields

$$
F(0,0)=\frac{1}{3}
$$

so that

$$
F(x, 0)=\frac{1}{3}+\frac{x}{6}, \quad x \in \mathbb{C} .
$$

Introducing now (30) and (31) into (24) and using the symmetry property (13), yields

$$
F(x, y)=\frac{2+x+y-x y^{2}-x^{2} y-2 x^{2} y^{2}}{6(1-x y)}=2 x y+x+y+2, \quad x \in \mathbb{C}, y \in \mathbb{C} .
$$

From (31) we find

$$
\left.\frac{d}{d x} F(x, 0)\right|_{x=1}=\frac{1}{6}
$$

which agrees with (20) when $k \rightarrow \infty$, and $\left.\frac{d^{2}}{d x^{2}} F(x, 0)\right|_{x=1}=0$ which implies from (23) that

$$
\operatorname{var}(N)=\frac{2}{3}
$$

when $k \rightarrow \infty$.

\section{$5 \quad$ Finite number of users}

In this section we solve the functional equation (11) when $k$ is finite with $k>3$ (stability condition). Throughout, for any complex number $z, \bar{z}$ denotes its complex conjugate and $\Re(z)$ its real part. 


\subsection{Preliminary results}

Define the set

$$
\mathcal{L}=\left\{w \in \mathbb{C},|w| \leq 1: w|w|^{2}+\bar{w}|w|^{2}-k|w|^{2}+k-2=0\right\} .
$$

\section{Lemma 1 (The contour $\mathcal{L}$ )}

$\mathcal{L}$ is a smooth closed contour that does not intersect with itself, is symmetric with respect to the real axis, and has the representation

$$
\mathcal{L}=\left\{w \in \mathbb{C},|w| \leq 1: w=\rho(\theta) e^{i \theta},-\pi \leq \theta \leq \pi\right\}
$$

with $\rho(\theta)$ the unique zero in $(0,1]$ of the polynomial in the variable $\rho$ defined by $2 \rho^{3} \cos \theta-k \rho^{2}+k-2$.

The function $\rho(\theta)$ is periodic with period $2 \pi$ and is infinitely differentiable on $\mathbb{R}, \rho(\theta)=\rho(-\theta)$ for all $\theta$, and

$$
\rho(0)=1, \quad \rho^{\prime}(0)=0, \quad \rho^{\prime \prime}(0)=\frac{1}{3-k}, \quad \rho^{\prime \prime \prime}(0)=0 .
$$

The proof is in Appendix D.

Let $\gamma$ be the conformal mapping of the unit disk $\mathcal{D}:=\{z \in C:|z|<1\}$ onto the interior of the contour $\mathcal{L}$, denoted by $\mathcal{L}^{+}$. This mapping is uniquely determined by the conditions $\gamma(0)=0$ and $\frac{d}{d z} \gamma(z)>0$ at $z=0$ [26, Theorem 1.2, 1.3]. By Theorem 2.24 in [26], the conformal mapping $\gamma(z)$ is continuous in the region $\{z \in \mathbb{C}:|z| \leq 1\}$, and maps the unit circle $\mathcal{C}$ one-to-one onto the contour $\mathcal{L}$. Moreover, the symmetry of $\mathcal{L}$ leads to the following property: for $|z| \leq 1$,

$$
\gamma(\bar{z})=\overline{\gamma(z)}
$$

The one-to-one conformal mapping from $\mathcal{C}$ onto $\mathcal{L}$ is given by

$$
\gamma(t)=\rho(\phi(\theta)) e^{i \phi(\theta)}
$$

for $t=e^{i \theta}$, where $\phi(\theta)$ is the unique continuous and strictly increasing mapping in $[-\pi, \pi]$ solution of the integral equation

$$
\phi(\theta)=\theta-\frac{1}{2 \pi} \int_{-\pi}^{\pi} \log (\rho(\phi(u))) \cot \left(\frac{u-\theta}{2}\right) d u, \quad \theta \in[-\pi, \pi] .
$$

Note that (39) can be rewritten as

$$
\phi(\theta)=\theta-\frac{1}{2 \pi} \int_{-\pi}^{\pi}\left(\log (\rho(\phi(u)))-\log (\rho(\phi(\theta))) \cot \left(\frac{u-\theta}{2}\right) d u, \quad \theta \in[-\pi, \pi]\right.
$$

since $\forall \theta \in[-\pi, \pi]$ the singular integral $\int_{-\pi}^{\pi} \cot \left(\frac{u-\theta}{2}\right) d u$ is equal to 0 in the sense of Cauchy principal value [11, Chapter I.1.5.] (Hint: $2 \log \left(\sin \left(\frac{u-\theta}{2}\right)\right)$ is a primitive of $\cot \left(\frac{u-\theta}{2}\right)$ yielding $\lim _{\epsilon \rightarrow 0}\left(\int_{\pi}^{\theta-\epsilon} \cot \left(\frac{u-\theta}{2}\right) d \theta\right.$ $\left.\left.+\int_{\theta+\epsilon}^{\pi} \cot \left(\frac{u-\theta}{2}\right) d \theta\right)=0\right)$. 
Since $-\phi(-\theta)$ is also a solution of (39) the uniqueness of the solution implies that for all $\theta \in[-\pi, \pi]$

$$
\phi(\theta)=-\phi(-\theta) .
$$

In particular (41) implies that $\phi(0)=0$ which in turn yields

$$
\gamma(1)=1
$$

from (38) together with $\rho(0)=1$ (cf. Lemma 1). Also, it is a simple exercise to show that $\phi(-\pi)=-\pi$ and $\phi(\pi)=\pi$ (Hint: $\cot \left(\frac{u \pm \pi}{2}\right)=-\tan (u / 2)$ and $\rho(\phi(u))=\rho(\phi(-u))$ from $(41)$ and $\left.\rho(\theta)=\rho(-\theta)\right)$.

The conformal mapping from $\mathcal{D}$ onto $\mathcal{L}^{+}$is given by

$$
\gamma(t)=\frac{1}{2 \pi i} \int_{\mathcal{C}} \frac{\gamma(u)}{u-t} d u=\frac{1}{2 \pi} \int_{-\pi}^{\pi} \frac{\rho(\phi(\theta))}{e^{i \theta}-t} e^{i \phi(\theta)+i \theta} d \theta, \quad|t|<1 .
$$

The part between (38)-(43) is taken from [16, Chapter II] (see also [6, Chapters I.6 and IV.3]).

We will also need the inverse of the conformal mapping $\gamma$, called $\gamma_{0}$. Fortunately the mapping $\gamma_{0}$ can be obtained in explicit form from [7, Section 7]. To this end, note that the contour $L(1,0)$ in $[7$, Eq. (7)] reduces to the contour $\mathcal{L}$ in (35) upon setting $\beta(s)=\frac{k-2}{k-2+s}$ and $\alpha=\frac{1}{2}$ so that the constant $a$ in $[7$, Eq. (5)]) is given by $a=\frac{2}{k-2}$. Then, by [7, Section 7]

$$
\gamma_{0}(w)=1-\frac{2 \delta}{w} \frac{(1-w)^{2}}{(1-\delta)^{2}}\left(\frac{k-2-w}{k-2-\delta}\right)\left[1+\frac{1}{\delta}\left(\frac{w-\delta}{1-w}\right) \sqrt{\frac{k-2-w \delta^{2}}{k-2-w}}\right], \quad \text { for } w \in \mathcal{L},
$$

with $\delta:=\frac{k-2-\sqrt{(k-2)^{2}+8(k-2)}}{4}$, and for $w \in \mathcal{L}^{+}$

$$
\gamma_{0}(w)=\frac{1}{2 \pi i} \int_{\mathcal{L}} \frac{\gamma_{0}(u)}{u-w} d u
$$

On the other hand, the r.h.s. of (44) can be analytically continued up to the region $\{w \in \mathbb{C}:|w|<k-2\}$, which shows (in particular) that $\gamma_{0}(w)$ is analytic for $|w|<1$ and continuous for $|w| \leq 1$. The mapping $\gamma_{0}$ is given by (45) for $w \in \mathcal{L}^{+}$and by (44) for $w \in\{w \in C:|w| \leq 1\}-\mathcal{L}^{+}$. This property will be used in the proof of Proposition 1.

Last

$$
\gamma_{0}(0)=0 \quad \text { and } \quad \gamma_{0}(1)=1
$$

which follows from $\gamma(0)=0, \gamma(1)=1$ and $\gamma_{0}(\gamma(t))=t$ for $t \in \mathcal{C}^{+} \cup \mathcal{C}$.

The contour $\mathcal{L}$ is depicted in Figure 3 for $k=4$ and $k=50$. Not surprisingly we note that as $k$ becomes large the contour $\mathcal{L}$ becomes close to the unit circle. This result follows from the fact that for all $\theta, \rho(\theta) \rightarrow 1$ as $k \rightarrow \infty$ (Hint: write the polynomial $Q(\rho):=2 \rho^{3} \cos \theta-k \rho^{2}+k-2$ as $Q(\rho)=k\left(2 \rho^{3} \cos \theta / k-\rho^{2}+1-2 / k\right)$; for any $\theta$, as $k \rightarrow \infty$ the polynomial $Q(\rho)$ may vanish only when $\rho \rightarrow 1$ ), which in turn implies from (39) that $\phi(\theta) \rightarrow \theta$. 


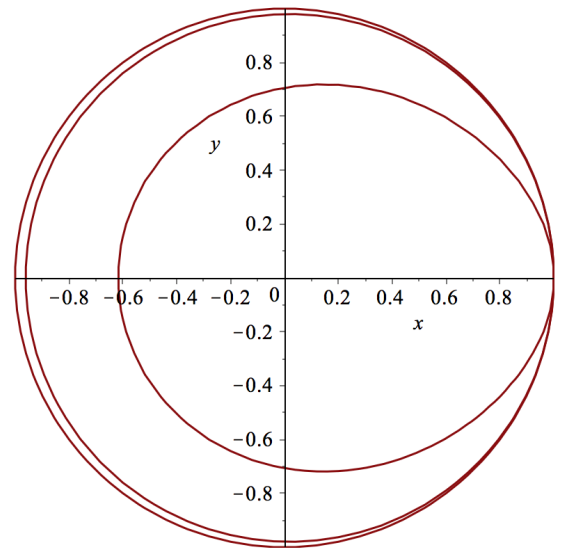

Figure 3: Inner contour is $\mathcal{L}$ for $k=4$, middle contour is $\mathcal{L}$ for $k=50$ and outer contour is the unit circle.

\subsection{Reduction of (11) to a boundary value problem}

Take $w \in \mathcal{L}$ and notice that $K(w, \bar{w})=0$. Since $F(w, \bar{w})$ is well-defined for $w \in \mathcal{L}$ (since $|w| \leq 1$ and $|\bar{w}| \leq 1$ when $w \in \mathcal{L})$ the left-hand side of $(11)$ must vanish when $(x, y)=(w, \bar{w})$. This generates the relation

$$
\left(1-\bar{w}|w|^{2}\right) F(w, 0)+\left(1-w|w|^{2}\right) F(0, \bar{w})+\left(\Re(w)|w|^{2}-1\right) F(0,0)=0, \quad \text { for } w \in \mathcal{L}
$$

Because $1-\bar{w}|w|^{2}=0$ if and only if $w=1$, we can divide both sides of (47) by $1-\bar{w}|w|^{2}$ when $w \neq 1$ to obtain

$$
F(w, 0)=-\left(\frac{1-w|w|^{2}}{1-\bar{w}|w|^{2}}\right) F(0, \bar{w})+\left(\frac{1-\Re(w)|w|^{2}}{1-\bar{w}|w|^{2}}\right) F(0,0), \quad \text { for } w \in \mathcal{L}-\{1\} .
$$

On the other hand

$$
\lim _{\substack{w \rightarrow 1 \\ w \in \mathcal{L}}} \frac{1-w|w|^{2}}{1-\bar{w}|w|^{2}}=\lim _{\theta \rightarrow 0} \frac{1-\rho(\theta)^{3}(\cos \theta+i \sin \theta)}{1-\rho(\theta)^{3}(\cos \theta-i \sin \theta)}=-1,
$$

by L'Hôpital's rule (Hint: $\rho(0)=1$ and $\left.\frac{d}{d \theta} \rho(\theta)\right|_{\theta=0}=0$ by Lemma 1 ), and

$$
\lim _{\substack{w \rightarrow 1 \\ w \in \mathcal{L}}} \frac{1-\Re(w)|w|^{2}}{1-\bar{w}|w|^{2}}=\lim _{\theta \rightarrow 0} \frac{1-\rho(\theta)^{3} \cos \theta}{1-\rho(\theta)^{3}(\cos \theta-i \sin \theta)}=0,
$$

by again using L'Hôpital's rule. This shows that the coefficients of $F(w, 0)$ and $F(0, \bar{w})$ in $(48)$ are well-defined when $w=1$, which implies that (49) can be analytically continued up to $w=1$, to give

$$
F(w, 0)=-\left(\frac{1-w|w|^{2}}{1-\bar{w}|w|^{2}}\right) F(0, \bar{w})+\left(\frac{1-\Re(w)|w|^{2}}{1-\bar{w}|w|^{2}}\right) F(0,0), \quad \text { for } w \in \mathcal{L} .
$$


Take $|t|=1$. Letting $w=\gamma(t)$ in (49) and using $\bar{w}=\gamma(1 / t)$ by (37) gives

$$
F(\gamma(t), 0)=-G(t) F(0, \gamma(1 / t))+g(t) F(0,0), \quad \forall|t|=1,
$$

where

$$
\begin{aligned}
G(t) & :=-\frac{1-\gamma(t)|\gamma(t)|^{2}}{1-\overline{\gamma(t)}|\gamma(t)|^{2}}, \\
g(t) & :=\frac{1-\Re(\gamma(t))|\gamma(t)|^{2}}{1-\overline{\gamma(t)}|\gamma(t)|^{2}} .
\end{aligned}
$$

A key observation is that $F(\gamma(t), 0)$ is regular for $|t|<1$ and $F(0, \gamma(1 / t))$ is regular for $|t|>1$ since $\mathcal{L}^{+} \subset\{z \in \mathbb{C}:|z|<1\}$. As a result, (50) defines a non-homogeneous Hilbert Boundary Value Problem (BVP) on $\mathcal{C}[11,14,17,27]$, which can be solved provided the functions $G$ and $g$ fulfill certain conditions (see proof of Proposition 1).

The technique of reducing the solution of certain two-dimensional functional equations (Eq. (11) in our case) to the solution of a boundary value problem (typically Riemann-Hilbert or Dirichlet problem) whose solution is known in closed-form - is due to Fayolle and Iasnogorodski [15] for polynomial kernels (in (11) the kernel is the coefficient of $F(x, y)$, namely, $K(x, y)$ ), and to Cohen and Boxma [10, 11] and Blanc [6] for Poisson kernels (i.e. kernels of the type $x y-r \beta\left(\lambda\left(1-r_{1} x-r_{2} y\right)\right),|r| \leq 1, \lambda>0, r_{1} \geq 0, r_{2} \geq 0$, $r_{1}+r_{2} \leq 1$, where $\beta(s)$ is the Laplace-Stieltjes transform of some probability distribution on $\left.[0, \infty)\right)$ via the use of uniformization techniques. Other related works include $[2,7,8,13,14]$ (non-exhaustive list).

Recall that $\arg [\varphi(z)]_{\mathcal{C}}$ denotes the variation of the argument of $\varphi(z)$ when $z$ describes the unit circle. Introduce

$$
\chi:=\frac{1}{2 \pi} \arg [G(t)]_{\mathcal{C}} \in \mathbb{Z},
$$

the index of the BVP defined in (50). The integer number $\chi$ gives the increment (positive or negative) of the argument of $G(t)$ when $t$ describes the unit circle once.

Define

$$
\begin{aligned}
I_{1} & :=\exp \left(\frac{1}{2 \pi i} \int_{\mathcal{C}} \frac{\log \left(u^{-1} G(u)\right)}{u-1} d u\right), \\
I_{2} & :=\exp \left(\frac{1}{2 \pi i} \int_{\mathcal{C}} \frac{\log \left(u^{-1} G(u)\right)}{u} d u\right), \\
I_{3} & :=\frac{1}{2 \pi i} \int_{\mathcal{C}} \frac{g(u) e^{-H(u)}}{u-1} d u, \\
I_{4} & :=\frac{1}{2 \pi i} \int_{\mathcal{C}} \frac{g(u) e^{-H(u)}}{u} d u .
\end{aligned}
$$

It is shown in Appendix E that $I_{2}=1$.

Proposition 1 below gives $F(x, 0)$ for $x \in \mathcal{L}^{+} \cup \mathcal{L}$. The function $F(0, x)$ for $x \in \mathcal{L}^{+} \cup \mathcal{L}$ is deduced from it since $F(x, 0)=F(0, x)$ for all $|x| \leq 1$. 
Proposition $1\left(F(x, 0)\right.$ for $\left.x \in \mathcal{L}^{+} \cup \mathcal{L}\right)$

For $x \in \mathcal{L}^{+}$

$$
F(x, 0)=e^{\frac{1}{2 \pi i}} \int_{\mathcal{C}} \frac{\log \left(u^{-1} G(u)\right)}{u-\gamma_{0}(x)} d u\left(\frac{F(0,0)}{2 \pi i} \int_{\mathcal{C}} \frac{g(u) e^{-H(u)}}{u-\gamma_{0}(x)} d u+a_{0}+a_{1} \gamma_{0}(x)\right),
$$

and for $x_{0} \in \mathcal{L}$

$$
\begin{aligned}
F\left(x_{0}, 0\right):= & \lim _{\substack{x \rightarrow x_{0} \\
x \in \mathcal{L}^{+}}} F(x, 0)=e^{H\left(t_{0}\right)}\left(\frac{F(0,0)}{2} g\left(\gamma_{0}\left(x_{0}\right)\right) e^{-H\left(\gamma_{0}\left(x_{0}\right)\right)}+\frac{F(0,0)}{2 \pi i} \int_{\mathcal{C}} \frac{g(u) e^{-H(u)}}{u-\gamma_{0}\left(x_{0}\right)} d u\right. \\
& \left.+a_{0}+a_{1} \gamma_{0}(x)\right),
\end{aligned}
$$

where $G(t)$ and $g(t)$ are given in (51) and (52), $\gamma_{0}(x)$ is given in (44) (resp. (45)) for $x \in \mathcal{L}$ (resp. $\left.x \in \mathcal{L}^{+}\right)$, and

$$
H(t):=\frac{1}{2} \log \left(t^{-1} G(t)\right)+\frac{1}{2 \pi i} \int_{\mathcal{C}} \frac{\log \left(u^{-1} G(u)\right)}{u-t} d u, \quad t \in \mathcal{C} .
$$

The constants $F(0,0), a_{0}$, and $a_{1}$ in (58)-(59) are given by

$$
\begin{aligned}
F(0,0) & =\Delta^{-1} \frac{2(k-3)}{3(k-2)} I_{2}, \\
a_{0} & :=\Delta^{-1} \frac{2(k-3)}{3(k-2)}\left(1-I_{2} I_{4}\right), \\
a_{1} & :=F(0,0),
\end{aligned}
$$

with $\Delta:=2 I_{1} I_{2}\left(I_{3}-I_{4}\right)+2 I_{1}\left(1+I_{2}\right)-I_{2}$.

Also,

$$
F(1,0)=\Delta^{-1} \frac{2(k-3)}{3(k-2)} I_{1}\left(I_{2}\left(I_{3}-I_{4}\right)+I_{2}+1\right) .
$$

Proof. It is shown in Lemma 4 in Appendix G that the index $\chi$ (defined in (53)) of the BVP defined (50) is equal to 1 . For $t \in \mathcal{C}$, define

$$
H(t)=\frac{1}{2} \log \left(t^{-1} G(t)\right)+\frac{1}{2 \pi i} \int_{\mathcal{C}} \log \left(u^{-1} G(u)\right) \frac{d u}{u-t} .
$$

It is shown in Lemma 3 in Appendix $\mathrm{F}$ that $G(t) \neq 0$ for $t \in \mathcal{C}$ and that the mappings $\log \left(t^{-1} G(t)\right)$, $g(t)$ and $g(t) e^{-H(t)}$ satisfy a Hölder condition on $\mathcal{C}$ (as continuous functions on this contour). Hence, the 
solution of the BVP in (50) is given by [11, Part I.2], [14, Chapter 5], [17, Chapter II], [27, Chapter 5]

$$
\begin{aligned}
F(\gamma(t), 0) & =e^{\Gamma(t)}(\Psi(t)+P(t)), \quad|t|<1, \\
F(0, \gamma(1 / t)) & =t^{-1} e^{\Gamma(t)}(\Psi(t)+P(t)), \quad|t|>1, \\
\lim _{\substack{t \rightarrow t_{0} \\
t \in \mathcal{C}^{+}}} F(\gamma(t), 0) & =e^{\Gamma^{+}\left(t_{0}\right)}\left(\Psi^{+}\left(t_{0}\right)+P\left(t_{0}\right)\right), \quad\left|t_{0}\right|=1, \\
\lim _{\substack{t \rightarrow t_{0} \\
t \in \mathcal{C}^{-}}} F(0, \gamma(1 / t)) & =t_{0}^{-1} e^{\Gamma^{-}\left(t_{0}\right)}\left(\Psi\left(t_{0}\right)^{-}+P\left(t_{0}\right)\right), \quad\left|t_{0}\right|=1,
\end{aligned}
$$

with $P(t)=a_{0}+a_{1} t$ a polynomial of degree one, and

$$
\begin{aligned}
\Psi(t) & :=\frac{F(0,0)}{2 \pi i} \int_{\mathcal{C}} g(u) e^{-H(u)} \frac{d u}{u-t}, \quad|t| \neq 1, \\
\Psi^{+}(t) & :=\frac{1}{2} F(0,0) g(t) e^{-H(t)}+\Psi(t), \quad|t|=1, \\
\Psi^{-}(t) & :=-\frac{F(0,0)}{2} g(t) e^{-H(t)}+\Psi(t), \quad|t|=1, \\
\Gamma(t) & :=\frac{1}{2 \pi i} \int_{\mathcal{C}} \log \left(u^{-1} G(u)\right) \frac{d u}{u-t}, \quad|t| \neq 1, \\
\Gamma^{+}\left(t_{0}\right) & :=\lim _{\substack{t \rightarrow t_{0} \\
|t|<1}} \Gamma(t)=\frac{1}{2} \log \left(t_{0}^{-1} G(t)\right)+\Gamma\left(t_{0}\right)=H\left(t_{0}\right), \quad\left|t_{0}\right|=1, \\
\Gamma^{-}\left(t_{0}\right) & :=\lim _{\substack{t \rightarrow t_{0} \\
|t|>1}} \Gamma(t)=-\frac{1}{2} \log \left(t_{0}^{-1} G\left(t_{0}\right)\right)+\Gamma\left(t_{0}\right), \quad|t|=1 .
\end{aligned}
$$

Letting $t=\gamma_{0}(x)$ in (65) and in (67) we obtain (58) and (59), respectively. Note that the singular integrals in (59) and (60) are defined in the sense of their Cauchy principal value [11, Section I.1.5.].

It remains to find the unknown constants $F(0,0), a_{0}$, and $a_{1}$. Letting $x=0$ in (58) and $x=1$ in (59) and using the identities $\gamma_{0}(0)=0$ and $\gamma_{0}(1)=1$ (cf. (46)), $G(1)=1$, and $g(1)=0$ (cf. Lemma 3 ) gives the equations

$$
\begin{aligned}
\left(1-I_{2} I_{4}\right) F(0,0)-I_{2} a_{0} & =0, \\
F(1,0)-I_{1} I_{3} F(0,0)-I_{1} a_{0}-I_{1} a_{1} & =0 .
\end{aligned}
$$

Two additional equations are needed to find the unknowns $a_{0}, a_{1}, F(0,0)$, and $F(1,0)$. One is provided by (15), namely,

$$
2 F(1,0)-F(0,0)=\frac{2(k-3)}{3(k-2)}
$$

Another one comes from (66). Letting $t \rightarrow \infty$ in (66) and using $\gamma(0)=0$ generates the equation

$$
F(0,0)=a_{1} .
$$


Letting $a_{1}=F(0,0)$ in (70) implies that Eqs (69)-(71) define a linear system of three equations with three unknowns $F(0,0), a_{0}$, and $F(1,0)$, whose unique solution is given in (61), (62) and (64). This concludes the proof.

The functional equation (11) and Proposition 1 yield the following corollary:

Corollary 1 (Solution of (11) in $\mathcal{L}^{+} \cup \mathcal{L} \times \mathcal{L}^{+} \cup \mathcal{L}$ )

For $x, y \in \mathcal{L}^{+} \cup \mathcal{L}$,

$$
F(x, y)=\frac{k-2}{x^{2} y+x y^{2}-k x y+k-2}\left(\left(1-x y^{2}\right) F(x, 0)+\left(1-x^{2} y\right) F(0, y)+\left(\frac{x^{2} y+x y^{2}}{2}-1\right) F(0,0)\right),
$$

where $F(x, 0)$ is given in (58) (resp. (59)) for $x \in \mathcal{L}^{+}$(resp. $x \in \mathcal{L}$ ) and $F(0, y)=F(y, 0)$ for all $y \in \mathcal{L}^{+} \cup \mathcal{L}$.

In particular, the r.h.s. of (73) gives $F(x, y)$ for all $x, y \in[0,1]$ as $[0,1] \subset \mathcal{L}^{+} \cup \mathcal{L}$.

Because $F(x, 0)$ is not analytic for $x \in \mathcal{L}$ due to the presence of the terms $\frac{1-\Re(x)|x|^{2}}{1-\bar{x}|x|^{2}}$ and $\frac{1-x|x|^{2}}{1-\bar{x}|x|^{2}}$ in $(59)$, the r.h.s. of (73) cannot be analytically extended to the entire region $\{x \in \mathbb{C}, y \in \mathbb{C}:|x| \leq 1,|y| \leq 1\}$, meaning that we do not have an explicit expression for $F(x, y)$ when $(x, y) \in\{x \in \mathbb{C}, y \in \mathbb{C}:|x| \leq 1,|y| \leq$ $1\}-\left\{x \in \mathbb{C}, y \in \mathbb{C}: x \in \mathcal{L}^{+} \cup \mathcal{L}, y \in \mathcal{L}^{+} \cup \mathcal{L}\right\}$. Fortunately, since the performance metrics of interest can be derived from $F(x, y)$ when $x, y \in[0,1]$ (see Section 7 ), not knowing $F(x, y)$ in explicit form in the entire region $\{x \in \mathbb{C}, y \in \mathbb{C}:|x| \leq 1,|y| \leq 1\}$ is not problematic.

Remark 1 When the kernel of the two-dimensional functional equation is a polynomial of degree two in each variable, as is the case for $K(x, y)$ in (12), the standard approach (cf., e.g., [15, 2] and references therein) is first to find the algebraic solution $y=Y(x)$ (resp. $x=X(y)$ ) of the equation $K(x, Y(x))=0$ (resp. $K(X(y), y)=0)$. From (12) we find

$$
Y(x)=\frac{x(k-x) \pm \sqrt{x\left(x(x-k)^{2}-4(k-2)\right)}}{2 x}, \quad x \in \mathbb{C} .
$$

$Y(x)$ has four branch real points inside the unit circle given by the four zeros of the discriminant $x(x(x-$ $\left.k)^{2}-4(k-2)\right)$, two zeros in $[0,1)$ and two zeros in $(1, \infty)$. The branch points in $[0,1)$ are $a_{1}=0$ and $a_{2}=\frac{k+2-\sqrt{(k-2)(k+6)}}{2}$. Similar results hold for $X(y)$ since $K(x, y)=K(y, x)$. Now, and this is the second step of the standard approach, when $x$ swipes twice the segment $\left[0, a_{2}\right], Y(x)$ describes a contour on which a boundary value problem can be set to calculate $F(x, y)$. Here, however, this contour goes to infinity since $Y(x) \rightarrow \infty$ as $x \rightarrow 0$ which yields a number of complications. This is in contrast with the vast majority of related works in which the contour is closed for polynomial kernels. The uniformization technique used to derive (50) allowed us to set a boundary value problem on a closed contour. Uniformization techniques are used when the kernel is not known explicitly (see [11, 6]); the present paper shows their usefulness for polynomial kernels. 


\section{Variance of the number of stored qubits at the switch}

This section is devoted to the calculation of the unknown term $\left.\frac{d^{2}}{d x^{2}} F(x, 0)\right|_{x=1}$ in the formula for the variance in (23). To this end, we will use (58) in Proposition 1. However, this calculation requires care as both integrals in the r.h.s. of (58) are principal value integrals as $x \rightarrow 1$. This is actually another specificity of the functional equation (11) (a first specificity was observed in Remark 1) as in general the point $x=1$ lies inside the contour on which the related BVP is defined (see Figure 3), so that calculating derivatives of $F(x, 0)$ at $x=1$ to get performance measures is not a problem (see, e.g., $[15,11,6,2]$ ).

For the sake of completeness we report below known results on Cauchy type integrals that we will use in this calculation. This material can be found in [11, Section I.1.], [17, Chapter 1], [27, Chapters 1, 2]. All results are stated for the unit circle $\mathcal{C}$ as this is what we need, but they hold for any smooth, non self-intersecting, and closed contour.

For any $t_{0} \in \mathcal{C}$ the singular integral $\frac{1}{2 \pi i} \int_{\mathcal{C}} \frac{d u}{u-t_{0}}$ exists in the sense of its Cauchy principal values ${ }^{1}[11$, p. 28, formula (5.4)], and is given by

$$
\frac{1}{2 \pi i} \int_{\mathcal{C}} \frac{d u}{u-t_{0}}=\frac{1}{2}
$$

Define $\Phi(t):=\frac{1}{2 \pi i} \int_{\mathcal{C}} \frac{\phi(u)}{u-t} d u$ for $t \notin \mathcal{C}$. If $\phi(t)$ satisfies a Hölder condition on $\mathcal{C}$ then the following so-called Plemelj-Sokhotski formulas hold

$$
\begin{aligned}
\Phi^{+}\left(t_{0}\right) & :=\lim _{\substack{t \rightarrow t_{0} \\
t \in \mathcal{C}^{+}}} \Phi(t)=\frac{1}{2} \phi\left(t_{0}\right)+\frac{1}{2 \pi i} \int_{\mathcal{C}} \frac{\phi(u)}{u-t} d u, \\
\Phi^{-}\left(t_{0}\right) & :=\lim _{\substack{t \rightarrow t_{0} \\
t \in \mathcal{C}^{-}}} \Phi(t)=-\frac{1}{2} \phi\left(t_{0}\right)+\frac{1}{2 \pi i} \int_{\mathcal{C}} \frac{\phi(u)}{u-t} d u .
\end{aligned}
$$

With the help of $(74)$ we can rewrite $\Phi^{+}\left(t_{0}\right)$ as (a similar formula holds for $\Phi^{-}\left(t_{0}\right)$, which we will not need)

$$
\Phi^{+}\left(t_{0}\right)=\phi\left(t_{0}\right)+\frac{1}{2 \pi i} \int_{\mathcal{C}} \frac{\phi(u)-\phi\left(t_{0}\right)}{u-t_{0}} d u
$$

If $\phi^{(m)}$, the $m$-th derivative of $\phi$ on $\mathcal{C}$, exists then for $t \notin \mathcal{C}$

$$
\begin{aligned}
\Phi^{(m)}(t):=\frac{d^{m}}{d t^{m}} \frac{1}{2 \pi i} \int_{\mathcal{C}} \frac{\phi(u)}{u-t} d u & =\frac{m !}{2 \pi i} \int_{\mathcal{C}} \frac{\phi(u)}{(u-t)^{m+1}} d u \\
& =\frac{1}{2 \pi i} \int_{\mathcal{C}} \frac{\phi^{(m)}(u)}{u-t} d u
\end{aligned}
$$

for $m=1,2, \ldots$; if in addition $\phi^{(m)}$ satisfies a Hölder condition on $\mathcal{C}$, the Plemelj-Sokhotski formula $(75)$

\footnotetext{
${ }^{1} \frac{1}{2 \pi i} \int_{\mathcal{C}} \frac{d u}{u-t_{0}}=\lim _{r \downarrow 0} \frac{1}{2 \pi i} \int_{\mathcal{C}-l} \frac{d u}{u-t_{0}}$ with $l$ is that part of $\mathcal{C}$ cut from $\mathcal{C}$ by a small circle with center at $t_{0}$ and radius $r$.
} 
applies, to give for $t_{0} \in \mathcal{C}$

$$
\begin{aligned}
{\left[\Phi^{(m)}\left(t_{0}\right)\right]^{+}:=\lim _{\substack{t \rightarrow t_{0} \\
t \in \mathcal{C}^{+}}} \Phi^{(m)}(t) } & =\frac{1}{2} \phi^{(m)}\left(t_{0}\right)+\frac{1}{2 \pi i} \int_{\mathcal{C}} \frac{\phi^{(m)}(u)}{u-t_{0}} d u \\
& =\phi^{(m)}\left(t_{0}\right)+\frac{1}{2 \pi i} \int_{\mathcal{C}} \frac{\phi^{(m)}(u)-\phi^{(m)}\left(t_{0}\right)}{u-t_{0}} d u
\end{aligned}
$$

for $m=1,2, \ldots$, where (81) follows from (74). Notice that the integrals in (77) and (81) are well-defined as Riemann improper integrals.

We are now in position to calculate $\left.\frac{d^{2}}{d x^{2}} F(x, 0)\right|_{x=1}$. For $t \in \mathcal{C}$, define

$$
\begin{aligned}
\alpha(t) & :=\log \left(t^{-1} G(t)\right), \\
\beta(t) & :=g(t) e^{-H(t)},
\end{aligned}
$$

where $G, g$, and $H$ are given in (51), (52), and (60), respectively. Introduce the integrals

$$
\begin{aligned}
I_{5} & :=\frac{1}{2 \pi i} \int_{\mathcal{C}} \frac{\alpha^{\prime}(u)-\alpha^{\prime}(1)}{u-1} d u, \\
I_{6} & :=\frac{1}{2 \pi i} \int_{\mathcal{C}} \frac{\beta^{\prime}(u)-\beta^{\prime}(1)}{u-1} d u, \\
I_{7} & :=\frac{1}{2 \pi i} \int_{\mathcal{C}} \frac{\alpha^{\prime \prime}(u)-\alpha^{\prime \prime}(1)}{u-1} d u, \\
I_{8} & :=\frac{1}{2 \pi i} \int_{\mathcal{C}} \frac{\beta^{\prime \prime}(u)-\beta^{\prime \prime}(1)}{u-1} d u .
\end{aligned}
$$

It is shown in Lemma 6 in Appendix $\mathrm{H}$ that the mappings $\alpha$ and $\beta$ are twice differentiable on $\mathcal{C}$ and that their second derivatives are continuous on $\mathcal{C}$, which shows that the integrals $I_{5}, \ldots, I_{8}$ are standard (and therefore finite) Riemann integrals.

Differentiating (58) w.r.t $x$ gives for $x \in \mathcal{C}^{+}\left(\right.$Hint: $a_{1}=F(0,0)$ )

$$
\begin{aligned}
& \frac{d}{d x} F(x, 0)=\frac{\gamma_{0}^{\prime}(x)}{2 \pi i} \int_{\mathcal{C}} \frac{\alpha(u)}{\left(u-\gamma_{0}(x)\right)^{2}} d u \times F(x, 0) \\
& \quad+e^{\frac{1}{2 \pi i} \int_{\mathcal{C}} \frac{\alpha(u)}{u-\gamma_{0}(x)} d u}\left(F(0,0) \frac{\gamma_{0}^{\prime}(x)}{2 \pi i} \int_{\mathcal{C}} \frac{\beta(u)}{\left(u-\gamma_{0}(x)\right)^{2}} d u+F(0,0) \gamma_{0}^{\prime}(x)\right), \\
& =\gamma_{0}^{\prime}(x)\left[\frac{F(x, 0)}{2 \pi i} \int_{\mathcal{C}} \frac{\alpha(u)}{\left(u-\gamma_{0}(x)\right)^{2}} d u+F(0,0) e^{\frac{1}{2 \pi i} \int_{\mathcal{C}} \frac{\alpha(u)}{u-\gamma_{0}(x)} d u}\left(\frac{1}{2 \pi i} \int_{\mathcal{C}} \frac{\beta(u)}{\left(u-\gamma_{0}(x)\right)^{2}} d u+1\right)\right], \\
& =\gamma_{0}^{\prime}(x)\left[\frac{F(x, 0)}{2 \pi i} \int_{\mathcal{C}} \frac{\alpha^{\prime}(u)}{u-\gamma_{0}(x)} d u+F(0,0) e^{\frac{1}{2 \pi i} \int_{\mathcal{C}} \frac{\alpha(u)}{u-\gamma_{0}(x)} d u}\left(\frac{1}{2 \pi i} \int_{\mathcal{C}} \frac{\beta^{\prime}(u)}{u-\gamma_{0}(x)} d u+1\right)\right],
\end{aligned}
$$


upon using twice (79) to establish (88). Note that (79) applies to the mappings $\alpha$ and $\beta$ since they are differentiable on $\mathcal{C}$, as mentioned above and proved in Lemma 6 in Appendix $\mathrm{H}$.

By using (79) and (81), the limit of (88) as $x \rightarrow 1$ is

$$
\left.\frac{d}{d x} F(x, 0)\right|_{x=1}=\gamma_{0}^{\prime}(1)\left[F(1,0)\left(\alpha^{\prime}(1)+I_{5}\right)+F(0,0) I_{1}\left(\beta^{\prime}(1)+I_{6}+1\right)\right] .
$$

We already know that $\left.\frac{d}{d x} F(x, 0)\right|_{x=1}=\frac{k}{6(k-2)}(\mathrm{cf} .(20))$.

Differentiating (88) w.r.t $x \in \mathcal{L}^{+}$, we obtain after using (79) (the latter result applies to mappings $\alpha^{\prime}$ and $\beta^{\prime}$ as they are differentiable on $\mathcal{C}$ - see Lemma 6)

$$
\begin{aligned}
& \frac{d^{2}}{d x^{2}} F(x, 0)=\frac{\gamma_{0}^{\prime \prime}(x)}{\gamma_{0}^{\prime}(x)} \times \frac{d}{d x} F(x, 0) \\
& \quad+\gamma_{0}^{\prime}(x)\left[\frac{d}{d x} F(x, 0) \times \frac{1}{2 \pi i} \int_{\mathcal{C}} \frac{\alpha^{\prime}(u)}{u-\gamma_{0}(x)} d u+F(x, 0) \frac{\gamma_{0}^{\prime}(x)}{2 \pi i} \int_{\mathcal{C}} \frac{\alpha^{\prime \prime}(u)}{u-\gamma_{0}(x)} d u\right. \\
& \quad+F(0,0) \frac{\gamma_{0}^{\prime}(x)}{2 \pi i} \int_{\mathcal{C}} \frac{\alpha^{\prime}(u)}{u-\gamma_{0}(x)} d u \times e^{\frac{1}{2 \pi i} \int_{\mathcal{C}} \frac{\alpha(u)}{u-\gamma_{0}(x)} d u}\left(\frac{1}{2 \pi i} \int_{\mathcal{L}} \frac{\beta^{\prime}(u)}{u-\gamma_{0}(x)} d u+1\right) \\
& \left.\quad+F(0,0) e^{\frac{1}{2 \pi i} \int_{\mathcal{C}} \frac{\alpha(u)}{u-\gamma_{0}(x)} d u} \times \frac{\gamma_{0}^{\prime}(x)}{2 \pi i} \int_{\mathcal{L}} \frac{\beta^{\prime \prime}(u)}{u-\gamma_{0}(x)} d u\right] .
\end{aligned}
$$

Finally, letting $x \rightarrow 1$ in (90) we find by using $\gamma_{0}(1)=1$, (79), and (81) with $t_{0}=1$, definitions (84)-(87), and $\left.\frac{d}{d x} F(x, 0)\right|_{x=1}=\frac{k}{6(k-2)}($ cf. (20)), that

$$
\begin{aligned}
\left.\frac{d^{2}}{d x^{2}} F(x, 0)\right|_{x=1}= & \frac{\gamma_{0}^{\prime \prime}(1)}{\gamma_{0}^{\prime}(1)} \times \frac{k}{6(k-2)}+\gamma_{0}^{\prime}(1)\left[\frac{k}{6(k-2)}\left(\alpha^{\prime}(1)+I_{5}\right)+F(1,0) \gamma_{0}^{\prime}(1)\left(\alpha^{\prime \prime}(1)+I_{7}\right)\right. \\
& \left.+F(0,0) \gamma_{0}^{\prime}(1) I_{1}\left\{\left(\alpha^{\prime}(1)+I_{5}\right)\left(\beta^{\prime}(1)+I_{6}+1\right)+\beta^{\prime \prime}(1)+I_{8}\right\}\right],
\end{aligned}
$$

where $F(0,0)$ is given in $(61)$ and $F(1,0)=\frac{1}{2} F(0,0)+\frac{2(k-3)}{6(k-2)}$ by $(15)$.

\section{$7 \quad$ Numerical results}

In this section, we address the numerical calculation of $F(0,0)$, the probability that the switch is empty, given in (61), and of the variance of the number of stored qubits at the switch, given in (23) and (91), when $3<k<\infty$.

\subsection{Steps in the numerical evaluation of $F(0,0)$ and $\operatorname{var}(N)$}

Throughout, $\theta \in[-\pi, \pi]$ and $u=e^{i \theta}$. Below are the steps to compute $F(0,0)$ and $\operatorname{var}(N)$. 
Step 1: Computation of $\alpha(u)$.

By (82) and (51)

$$
\alpha(u)=\log \left(e^{-i \theta} \tilde{G}(\phi(\theta))\right)
$$

with

$$
\tilde{G}(\theta):=-\frac{1-\rho(\theta)^{3} e^{i \theta}}{1-\rho(\theta)^{3} e^{-i \theta}}
$$

In (128), $\rho(\theta)$ is the only zero in $(0,1]$ of $2 \cos (\theta) \rho^{3}-k \rho^{2}+k-2$ (see Lemma 1$)$ and in $(92) \phi(\theta)$, defined in (39), is obtained as the output of the following iterative scheme:

$$
\begin{aligned}
\phi_{0}(\theta) & :=\theta \\
\phi_{n+1}(\theta) & :=\theta-\frac{1}{2 \pi} \int_{-\pi}^{\pi} \log \left(\rho\left(\phi_{n}(\omega)\right)\right) \cot \left(\frac{\omega-\theta}{2}\right) d \omega . \quad n=0,1, \ldots
\end{aligned}
$$

Step 2: Computation of $\alpha^{\prime}(u)$ and $\alpha^{\prime \prime}(u)$.

The mappings $\alpha^{\prime}(u)$ and $\alpha^{\prime \prime}(u)$ are given in (130)-(131), with $\tilde{G}^{\prime}(\theta)$ given in (134) and $\tilde{G}^{\prime \prime}(\theta)$ given in (137). The first and second derivatives of $\rho(\theta)$ showing up in (134) and (137) are obtained by differentiating the equation $2 \cos (\theta) \rho(\theta)^{3}-k \rho(\theta)^{2}+k=0$, which gives

$$
\begin{aligned}
\rho^{\prime}(\theta) & =\frac{\rho(\theta)^{2} \sin \theta}{3 \rho(\theta) \cos \theta-k}, \\
\rho^{\prime \prime}(\theta) & =\frac{\rho(\theta)^{2}}{3 \rho(\theta) \cos \theta-k}\left(\cos \theta+\frac{5 \rho(\theta) \sin (\theta)^{2}}{3 \rho(\theta) \cos \theta-k}-\frac{3 \rho(\theta)^{2} \sin (\theta)^{2} \cos \theta}{(3 \rho(\theta) \cos \theta-k)^{2}}\right),
\end{aligned}
$$

where we used (96) to obtain (97). The first and second derivatives of $\phi(\theta)$ in (130)-(131) could be obtained by differentiating the defining equation (39). However, since the mapping $\gamma_{0}$ is known explicitly (see (44)), it is more computationally efficient to differentiate the identity $\gamma_{0}(\gamma(w))=w$ with $w=\rho(\phi(\theta)) e^{i \phi(\theta)} \in \mathcal{L}$ (see Section 5.1), to obtain

$$
\phi^{\prime}(\theta)=\frac{i e^{i(\theta-\phi(\theta))}}{\rho^{\prime}(\phi(\theta))+i \rho(\phi(\theta))} \times \frac{1}{\gamma_{0}^{\prime}\left(\rho(\phi(\theta)) e^{i \phi(\theta)}\right)},
$$

and

$$
\begin{aligned}
& \phi^{\prime \prime}(\theta)=-\frac{e^{i(\theta-\phi(\theta))}}{\left(\rho^{\prime}(\phi(\theta))+i \rho(\phi(\theta))\right)^{2} \gamma_{0}^{\prime}\left(\rho(\phi(\theta)) e^{i \phi(\theta)}\right)^{2}} \\
& \times {\left[e^{i \theta} \phi^{\prime}(\theta)\left(-i \rho(\phi(\theta))^{2}-2 \rho^{\prime}(\phi(\theta)) \rho(\phi(\theta))+i \rho^{\prime}(\phi(\theta))^{2}\right) \gamma_{0}^{\prime \prime}\left(\rho(\phi(\theta)) e^{i \phi(\theta)}\right)\right.} \\
&\left.+\left(-2 \phi^{\prime}(\theta) \rho^{\prime}(\phi(\theta))+i \phi^{\prime}(\theta) \rho^{\prime \prime}(\phi(\theta))-i \phi^{\prime}(\theta) \rho(\phi(\theta))+i \rho(\phi(\theta))+\rho^{\prime}(\phi(\theta))\right) \gamma_{0}^{\prime}\left(\rho(\phi(\theta)) e^{i \phi(\theta)}\right)\right] .
\end{aligned}
$$


Step 3: Computation of $\beta(u)$.

By (83) and (52)

$$
\beta(u)=\tilde{g}(\phi(\theta)) e^{-H(u)},
$$

where $\tilde{g}$ is given in (129) and $H$ is given in (142).

Step 4: Computation of $\beta^{\prime}(u)$ and $\beta^{\prime \prime}(u)$.

The mappings $\beta^{\prime}(u)$ and $\beta^{\prime \prime}(u)$ are given in (132) and (133), respectively, with the mappings $H^{\prime}$ and $H^{\prime \prime}$ given in (143) and (144), respectively.

Step 5: Computation of integrals $I_{1}, I_{3}, \ldots, I_{8}$.

Since the integrands in $I_{5}, \ldots, I_{8}$ in $(84)$-(87) are well-defined on $\mathcal{C}$ these integrals can be computed without any problem by using (130)-(133). After easy but lengthy algebra constants $\alpha^{\prime}(1)$ in (84) and $\alpha^{\prime \prime}(1)$ in (86) have been found to be

$$
\alpha^{\prime}(1)=\phi^{\prime}(0) \frac{k}{k-3}-1 \quad \text { and } \quad \alpha^{\prime \prime}(1)=1-\phi^{\prime}(0) \frac{k}{k-3}+i \phi^{\prime \prime}(0) \frac{k}{k-3} .
$$

Constants $\beta^{\prime}(1)$ in $I_{6}$ and $\beta^{\prime \prime}(1)$ in $I_{8}$ are calculated from (132) and (133), respectively.

The integrand in $I_{1}$ in (54) is well-defined on $\mathcal{C}$ since $G(1)=0$ by Lemma 3 in Appendix F, so that it can be evaluated using (92) since $I_{1}$ can be rewritten as $I_{1}=\exp \left(\frac{1}{2 \pi} \int_{\mathcal{C}} \frac{\alpha(u)}{u-1} d u\right)$. The integrand in $I_{3}$ in (56) is also well-defined as $g(1)=0$ by Lemma 3 in Appendix F, and can be easily evaluated using (100) since $I_{3}$ can be rewritten as $I_{3}=\frac{1}{2 \pi i} \int_{\mathcal{C}} \frac{\beta(u)}{u-1} d u$. Last, $I_{4}$ in (57) is computed by using (100) since $I_{4}$ can be rewritten as $I_{4}=\frac{1}{2 \pi i} \int_{\mathcal{C}} \frac{\beta(u)}{u} d u$.

Steps 1-5 allow us to compute $F(0,0)$ in $(61)$ and $\operatorname{var}(N)$ in $(23)$ (recall that $I_{2}=1$ as shown in Appendix E).

\subsection{Plots $k \rightarrow\{F(0,0), \mathbb{E}[N], \operatorname{var}(N)\}$}

We recall that $N$ is the (stationary) number of qubits stored at the switch. The mappings $k \rightarrow F(0,0)=$ $P$ (empty system), $k \rightarrow \mathbb{E}[N]=\frac{k}{k-3}$ (see $(21)$ ), and $k \rightarrow \operatorname{var}(N)$ are displayed in Figures 4-6, respectively. On each figure the dotted line is the corresponding asymptotic result when $k=\infty(F(0,0)=1 / 3$ and $\operatorname{var}(N)=2 / 3-$ see Section 4 , and $\mathbb{E}[N]=1)$.

We observe that beyond a certain threshold for the number of users (say $k=40$ in each figure) the performance metrics do not evolve much. In contrast, below this threshold there are sharp variations in the performance metrics.

The program used to generate Figures 4 and 6 is available at https://github.com/gvardoyan/ TripartiteSwitch 




Figure 4: Solid line: Prob. switch memory is empty as a function of number of links $k$ for $k \geq 4$. Dotted line: $P($ empty system $)=\frac{1}{3}$ when $k=\infty-$ see $(30)$.

\section{Conclusion}

In this work, we analyzed a quantum entanglement distribution switch in which each client has a dedicated link connected to the switch. The switch serves tripartite entanglement to $k \geq 3$ users according to incoming requests. At the link level, entangled states are generated across each link according to independent Poisson processes with a constant and identical rate $(\mu)$, resulting in two-qubit maximallyentangled states. These qubits are stored at local quantum memories, one at the user and the other at the switch. When three link-level entangled stated are accrued, the switch performs three-qubit measurement (successful with probability $q$ ) to provide end-to-end entanglement to users of groups of size three. This system can also be seen as an assembly-like stochastic queueing system with one central node (or plant) receiving parts or components of a product from geographically distant manufacturing plants. As soon as three parts from three distinct plants are available at the central plant, they are assembled instantaneously and immediately leave the system. Under simplifying assumptions (identical links, infinite storage capabilities at the users and at the switch, no cut-off times at quantum storage) we have obtained the switch capacity, the probability that no there are no entangled qubits in the switch memory, the expected number of stored qubits at the switch and the variance of the number of stored qubits at the switch. These quantities are obtained by solving a two-dimensional equation satisfied by the generating function of a two-dimensional Markov chain modeling the state of the system. The solution of the functional equation is obtained by reducing it to a boundary value problem on a closed contour, which we solve. This analysis is of independent interest due to the form of the kernel of the functional equation (cf. Remark 1) along with the fact that point 1 lies on the contour (see comment at the beginning of Section 6) which generates some difficulties when computing some performance metrics. In terms of future work, it would be interesting to relax the assumption that quantum states are stored indefinitely, as cut-off times have been found to play an important role in controlling quantum state fidelity (quality) [32]. This could be done by assuming that these quantum states have an exponential lifetime with a constant rate. 


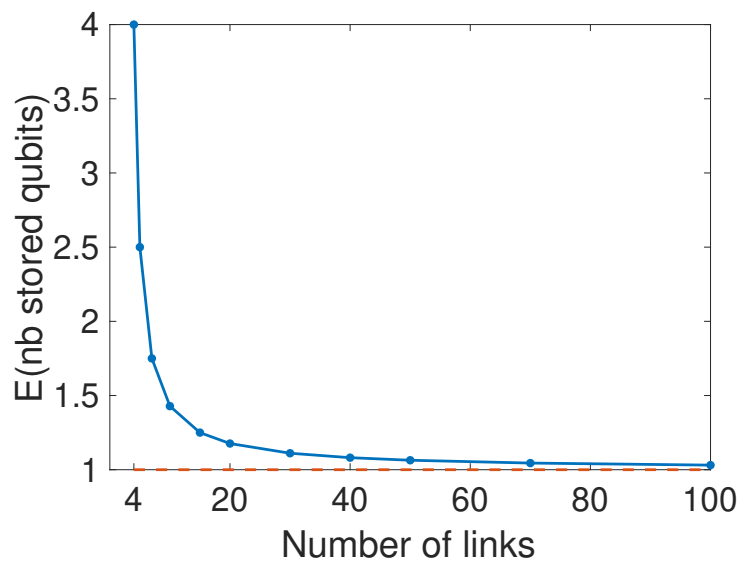

Figure 5: Solid line: Expected number of stored qubits at the switch as a function of number of links $k$ for $k \geq 4$. Dotted line: $\mathbb{E}[N]=1$ when $k \rightarrow \infty$ - see $(21)$.

\section{A Proof of (10)}

Note that $\pi(0,0)=F(0,0)$. We will need the following identities:

$$
\begin{aligned}
\sum_{\substack{i \geq 1 \\
j \geq 1}} \pi(i, j) x^{i} y^{j} & =F(x, y)-F(x, 0)-F(0, y)+F(0,0) \\
\sum_{\substack{i \geq 0 \\
j \geq 1}} \pi(i, j) x^{i} y^{j}= & F(x, y)-F(x, 0) \\
\sum_{\substack{i \geq 1 \\
j \geq 0}} \pi(i, j) x^{i} y^{j}= & F(x, y)-F(0, y) \\
\sum_{\substack{i \geq 2 \\
j \geq 2}} \pi(i, j) x^{i} y^{j}= & F(x, y)-F(x, 0)-F(0, y)+F(0,0)-y \sum_{i \geq 1} \pi(i, 1) x^{i}-x \sum_{j \geq 1} \pi(1, j) y^{j}+\pi(1,1) x y \\
\sum_{\substack{i \geq 3 \\
j \geq 3}} \pi(i, j) x^{i} y^{j}= & \sum_{\substack{i \geq 2 \\
j \geq 2}} \pi(i, j) x^{i} y^{j}-x^{2} \sum_{j \geq 2} \pi(2, j) y^{j}-y^{2} \sum_{i \geq 2} \pi(i, 2) x^{i}+x^{2} y^{2} \pi(2,2) \\
= & F(x, y)-F(x, 0)-F(0, y)+F(0,0)-y \sum_{i \geq 1} \pi(i, 1) x^{i} \\
- & x \sum_{j \geq 1} \pi(1, j) y^{j}-x^{2} \sum_{j \geq 2} \pi(2, j) y^{j}-y^{2} \sum_{i \geq 2} \pi(i, 2) x^{i}+x y \pi(1,1)+x^{2} y^{2} \pi(2,2)
\end{aligned}
$$




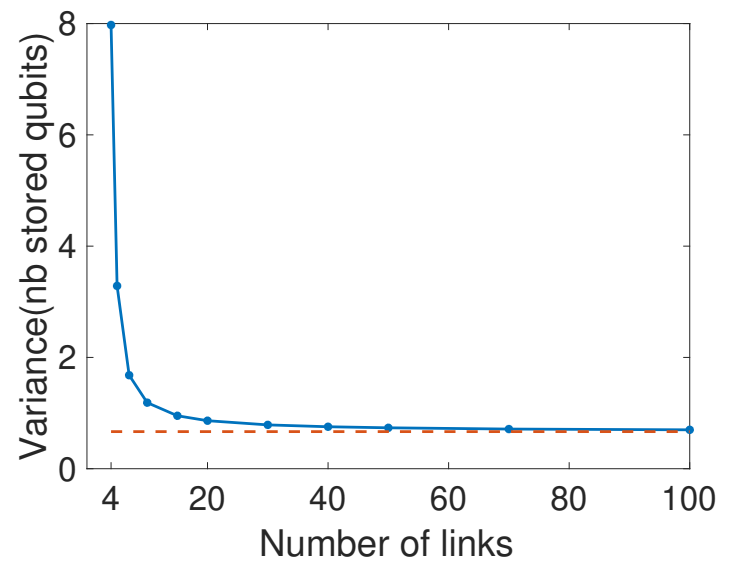

Figure 6: Solid line: Variance of number of stored qubits at the switch as a function of number of links $k$ for $k \geq 4$. Dotted line: $\operatorname{var}(N)=\frac{2}{3}$ as $k \rightarrow \infty-$ see $(33)$.

where the latter equality comes from (104). Multiplying both sides of Eq. (5) by $x^{i}$ and then summing over all $i \geq 2$ yields

$$
\begin{aligned}
(2 \lambda+\nu) \sum_{i \geq 2} \pi(i, 0) x^{i} & =\frac{\nu}{x} \sum_{i \geq 2} \pi(i+1,1) x^{i+1}+\lambda x \sum_{i \geq 2} \pi(i-1,0) x^{i-1} \\
& =\frac{\nu}{x} \sum_{i \geq 3} \pi(i, 1) x^{i}+\lambda x \sum_{i \geq 1} \pi(i, 0) x^{i} \\
& =\frac{\nu}{x}\left(\sum_{i \geq 1} \pi(i, 1) x^{i}-\pi(2,1) x^{2}-\pi(1,1) x\right)+\lambda x(F(x, 0)-\pi(0,0)) .
\end{aligned}
$$

Since $\sum_{i \geq 2} \pi(i, 0) x^{i}=F(x, 0)-\pi(1,0) x-\pi(0,0)$, we obtain the following from Eq. (106) by using (1) and $(2)$ :

$$
\frac{\nu}{x} \sum_{i \geq 1} \pi(i, 1) x^{i}=(\lambda+\lambda(1-x)+\nu) F(x, 0)-\frac{\nu x}{2} \pi(0,0), \quad|x| \leq 1,
$$

Similarly, from Eq. (6) we obtain by using (1) and (3)

$$
\frac{\nu}{y} \sum_{j \geq 1} \pi(1, j) y^{j}=(\lambda+\lambda(1-y)+\nu) F(0, y)-\frac{\nu y}{2} \pi(0,0), \quad|y| \leq 1 .
$$


Multiplying now both sides of Eq. (7) by $x^{i}$ and then summing over all $i \geq 2$, gives

$$
\begin{aligned}
(2 \lambda+\nu) \sum_{i \geq 2} \pi(i, 1) x^{i} & =\frac{\nu}{x} \sum_{i \geq 2} \pi(i+1,2) x^{i+1}+\lambda x \sum_{i \geq 2} \pi(i-1,1) x^{i-1}+(\lambda+\nu) \sum_{i \geq 2} \pi(i, 0) x^{i} \\
& =\frac{\nu}{x} \sum_{i \geq 3} \pi(i, 2) x^{i}+\lambda x \sum_{i \geq 1} \pi(i, 1) x^{i}+(\lambda+\nu) \sum_{i \geq 2} \pi(i, 0) x^{i} \\
& =\frac{\nu}{x}\left(\sum_{i \geq 1} \pi(i, 2) x^{i}-\pi(2,2) x^{2}-\pi(1,2) x\right)+\lambda x \sum_{i \geq 1} \pi(i, 1) x^{i} \\
& +(\lambda+\nu)\left(\sum_{i \geq 0} \pi(i, 0) x^{i}-\pi(1,0) x-\pi(0,0)\right)
\end{aligned}
$$

With $\sum_{i \geq 2} \pi(i, 1) x^{i}=\sum_{i \geq 1} \pi(i, 1) x^{i}-\pi(1,1) x$, we find for $|x| \leq 1$

$$
\begin{aligned}
\frac{\nu}{x} \sum_{i \geq 1} \pi(i, 2) x^{i} & =(\lambda+\lambda(1-x)+\nu) \sum_{i \geq 1} \pi(i, 1) x^{i}+\nu x \pi(2,2)+\nu \pi(1,2)-(\lambda+\nu) F(x, 0) \\
& +(\lambda+\nu) x \pi(1,0)+(\lambda+\nu) \pi(0,0)-(2 \lambda+\nu) x \pi(1,1) .
\end{aligned}
$$

Similarly for $|y| \leq 1$

$$
\begin{aligned}
\frac{\nu}{y} \sum_{j \geq 1} \pi(2, j) y^{j} & =(\lambda+\lambda(1-y)+\nu) \sum_{j \geq 1} \pi(1, j) y^{j}+\nu y \pi(2,2)+\nu \pi(2,1)-(\lambda+\nu) F(0, y) \\
& +(\lambda+\nu) y \pi(0,1)+(\lambda+\nu) \pi(0,0)-(2 \lambda+\nu) y \pi(1,1) .
\end{aligned}
$$

Multiplying now both sides of Eq. (9) by $x^{i} y^{j}$ and then summing over all $i \geq 2, j \geq 2$, yields

$$
\begin{aligned}
& (2 \lambda+\nu) \sum_{\substack{i \geq 2 \\
j \geq 2}} \pi(i, j) x^{i} y^{j} \\
& =\frac{\nu}{x y} \sum_{\substack{i \geq 2 \\
j \geq 2}} \pi(i+1, j+1) x^{i+1} y^{j+1}+\lambda x \sum_{\substack{i \geq 2 \\
j \geq 2}} \pi(i-1, j) x^{i-1} y^{j}+\lambda y \sum_{\substack{i \geq 2 \\
j \geq 2}} \pi(i, j-1) x^{i} y^{j-1}, \\
& =\frac{\nu}{x y} \sum_{\substack{i \geq 3 \\
j \geq 3}} \pi(i, j) x^{i} y^{j}+\lambda x \sum_{\substack{i \geq 1 \\
j \geq 2}} \pi(i, j) x^{i} y^{j}+\lambda y \sum_{\substack{i \geq 2 \\
j \geq 1}} \pi(i, j) x^{i} y^{j} .
\end{aligned}
$$


By using identities (101)-(105), Eq. (110) rewrites

$$
\begin{aligned}
& (2 \lambda+\nu)\left[F(x, y)-F(x, 0)-F(0, y)+F(0,0)-y \sum_{i \geq 1} \pi(i, 1) x^{i}-x \sum_{j \geq 1} \pi(1, j) y^{j}+\pi(1,1) x y\right] \\
& =\left(\lambda(x+y)+\frac{\nu}{x y}\right)[F(x, y)-F(x, 0)-F(0, y)+F(0,0)]-\left(\lambda x y+\frac{\nu}{x}\right) \sum_{i \geq 1} \pi(i, 1) x^{i} \\
& -\left(\lambda x y+\frac{\nu}{y}\right) \sum_{j \geq 1} \pi(1, j) y^{-} \frac{\nu y}{x} \sum_{i \geq 2} \pi(i, 2) x^{i}-\frac{\nu x}{y} \sum_{j \geq 2} \pi(2, j) y^{j}+\nu \pi(1,1)+\nu x y \pi(2,2) .
\end{aligned}
$$

Simple algebra in (111) gives

$$
\begin{aligned}
& \left(\lambda(1-x)+\lambda(1-y)+\nu\left(1-\frac{1}{x y}\right)\right)[F(x, y)-F(x, 0)-F(0, y)+F(0,0)] \\
& =\left((2 \lambda+\nu) y-\lambda x y-\frac{\nu}{x}\right) \sum_{i \geq 1} \pi(i, 1) x^{i}+\left((2 \lambda+\nu) x-\lambda x y-\frac{\nu}{y}\right) \sum_{j \geq 1} \pi(1, j) y^{j} \\
& -\quad \frac{\nu y}{x} \sum_{i \geq 1} \pi(i, 2) x^{i}-\frac{\nu x}{y} \sum_{j \geq 1} \pi(2, j) y^{j}-((2 \lambda+\nu) x y-\nu) \pi(1,1)+\nu y \pi(1,2)+\nu x \pi(2,1)+\nu x y \pi(2,2), \\
& =-\frac{\nu}{x} \sum_{i \geq 1} \pi(i, 1) x^{i}-\frac{\nu}{y} \sum_{j \geq 1} \pi(1, j) y^{j}+(\lambda+\nu) y F(x, 0)+(\lambda+\nu) x F(0, y) \\
& -\nu x y \pi(2,2)+((2 \lambda+\nu) x y+\nu) \pi(1,1)-(\lambda+\nu) x y(\pi(1,0)+\pi(0,1))-(\lambda+\nu)(x+y) \pi(0,0),
\end{aligned}
$$

where we have used (109) and (109) to derive the last equality. Using (107) and (108), we now obtain

$$
\begin{aligned}
\left(\lambda(1-x)+\lambda(1-y)+\nu\left(1-\frac{1}{x y}\right)\right)[F(x, y)-F(x, 0)-F(0, y)+F(0,0)] \\
=-(\lambda(1-x)+(\lambda+\nu)(1-y)) F(x, 0)-(\lambda(1-y)+(\lambda+\nu)(1-x)) F(0, y) \\
\quad-\left(\frac{2 \lambda+\nu}{2}\right)(x+y) \pi(0,0)+d(x, y)
\end{aligned}
$$

with

$$
d(x, y):=\nu \pi(1,1)+x y(-\nu \pi(2,2)+(2 \lambda+\nu) \pi(1,1)-(\lambda+\nu) \pi(1,0)-(\lambda+\nu) \pi(0,1)) .
$$

From (4) we see that the second term in the right-hand side of (113) is equal to zero, so that

$$
d(x, y)=\nu \pi(1,1)=(2 \lambda+\nu) \pi(0,0)
$$


where the second equality comes from (1). Collecting terms having $F(x, 0), F(0, y)$, and $F(0,0)$ as factors in (112) and using (114) finally yields

$$
\begin{aligned}
\left(\lambda(1-x)+\lambda(1-y)+\nu\left(1-\frac{1}{x y}\right)\right) F(x, y) & =\nu\left(y-\frac{1}{x y}\right) F(x, 0)+\nu\left(x-\frac{1}{x y}\right) F(0, y) \\
& -\nu\left(\frac{x+y}{2}-\frac{1}{x y}\right) F(0,0),
\end{aligned}
$$

which concludes the proof.

\section{B Zero of $K(x, y)$ for $|x|=1$}

Lemma 2 For $|x|=1$, the equation $K(x, y)=0$ as a unique solution $y=y(x)$ such that $|y(x)| \leq 1$. Moreover $y(1):=1, y^{\prime}(1)=-1$ and $y^{\prime \prime}(1)=\frac{2(k-2)}{k-3}$.

Proof. Recall that $k>3$.

Assume first that $x=1$. Since $K(1, y)=-(y-1)(y-(k-2))$ we see that $K(1, y)$ has a unique zero in the unit disk $\{|y| \leq 1\}$, located at $y=1$. Assume now that $x$ is fixed with $|x|=1$ and $x \neq 1$. Define $h(x, y)=x(k-x) y$. For $|y|=1$, we have

$$
|K(x, y)-h(x, y)|=\left|x y^{2}+k-2\right| \leq k-1<|k-x|=|x(k-x) y|=|h(x, y)|,
$$

where the second inequality holds since ${ }^{2}|x|=1$ with $x \neq 1$. By Rouché's theorem we conclude that $K(x, y)$ and $h(x, y)$ have the same number of zeros in the unit disk $\{|y|<1\}$. Since $h(x, y)$ has the only zero $y=0$ in $\{|y|<1\}, K(x, y)$ has a unique zero in $\{|y|<1\}$. This proves the first part of the lemma.

The fact that $y(x)$ has first and second derivatives at $x=1$ follows ${ }^{3}$ from the implicit function theorem [26], that applies here since $K(x, y)$ is analytic in $\mathbb{C} \times \mathbb{C}, K(1,1)=1$, and $\left.\frac{\partial}{\partial y} K(x, y)\right|_{(x, y)=(1,1)}=k-3 \neq 0$.

Differentiating $K(x, y(x))$ with respect to $x$ and letting $x=1$ gives $y^{(1)}(1)=-1$ by using $y(1)=1$. Differentiating twice $K(x, y(x))$ with respect to $x$ and letting $x=1$ gives $y^{\prime \prime}(1)=\frac{2(k-2)}{k-3}$ upon using $y(1)=1$ and $y^{\prime}(1)=-1$. This concludes the proof.

\section{Proof of (22)}

When $x=y(11)$ becomes

$$
\begin{aligned}
\left(2 x^{3}-k x^{2}+k-2\right) F(x, x) & =(k-2)\left(\left(1-x^{3}\right) F(x, 0)+\left(1-x^{3}\right) F(0, x)+\left(x^{3}-1\right) F(0,0)\right), \\
& =(k-2)\left(1-x^{3}\right)(2 F(x, 0)-F(0,0)),
\end{aligned}
$$

\footnotetext{
${ }^{2}$ To see that, take $x=e^{i \theta}$ with $x \in(0,2 \pi)$. Then, $|k-x|^{2}=k^{2}+1-2 k \cos (\theta)$ which is strictly larger than $\left|k-1^{2}\right|=$ $(k-1)^{2}=k^{2}-2 k+1$ when $\cos (\theta)<1$.

${ }^{3}$ Since $K(x, y)$ is analytic in $\mathbb{C} \times \mathbb{C}, K(1,1)=1$ and $\left.\frac{\partial}{\partial y} K(x, y)\right|_{(x, y)=(1,1)}=k-3 \neq 0$, there exists a neighborhood $\mathcal{V}$ of $x=1$ in the complex plane and an analytic function $w(x)$ defined on $\mathcal{V}$ such that $K(x, w(x))=0$ for all $x \in \mathcal{V}$ and $w(1)=1$. This necessarily implies that $w(x)=y(x)$ for all $x \in \mathcal{V} \cap\{|x|=1\}$, and therefore that $y(x)$ is indefinitely differentiable at $x=1$ since $1 \in \mathcal{V} \cap\{|x|=1\}$.
} 
since $F(x, 0)=F(0, x)$. From the identities $2 x^{3}-k x^{2}+k-2=(1-x)\left(-2 x^{2}+x(k-2)+k-2\right)$ and $1-x^{3}=(1-x)\left(1+x+x^{2}\right)$ the above becomes

$$
\left(-2 x^{2}+x(k-2)+k-2\right) F(x, x)=(k-2)\left(1+x+x^{2}\right)(2 F(x, 0)-F(0,0)),
$$

upon dividing both sides by $1-x$. Let us calculate the 2 nd-order derivative of the 1.h.s. of (115). We have

$$
\frac{d}{d x}\left(\left(-2 x^{2}+x(k-2)+k-2\right) F(x, x)\right)=(-4 x+k-2) F(x, x)+2\left(-2 x^{2}+x(k-2)+k-2\right) \frac{d}{d x} F(x, x),
$$

and

$$
\begin{aligned}
& \frac{d^{2}}{d x^{2}}\left(\left(-2 x^{2}+x(k-2)+k-2\right) F(x, x)\right) \\
& \quad=-4 F(x, x)+2(-4 x+k-2) \frac{d}{d x} F(x, x)+\left(-2 x^{2}+x(k-2)+k-2\right) \frac{d^{2}}{d x^{2}} F(x, x) .
\end{aligned}
$$

Letting $x=1$ gives

$$
\begin{aligned}
& \left.\frac{d^{2}}{d x^{2}}\left(\left(-2 x^{2}+x(k-2)+k-2\right) F(x, x)\right)\right|_{x=1} \\
& \quad=-4 F(1,1)+\left.2(k-6) \frac{d}{d x} F(x, x)\right|_{x=1}+\left.2(k-3) \frac{d^{2}}{d x^{2}} F(x, x)\right|_{x=1}, \\
& \quad=-4+\frac{2 k(k-6)}{k-3}+\left.2(k-3) \frac{d^{2}}{d x^{2}} F(x, x)\right|_{x=1},
\end{aligned}
$$

since $F(1,1)=1$ and $\left.\frac{d}{d x} F(x, x)\right|_{x=1}=\mathbb{E}[N]=\frac{k}{k-3}$ from $(21)$.

Similarly, let us calculate the 2nd-order derivative of the r.h.s. of (115). We have

$$
\begin{aligned}
& \frac{d}{d x}\left((k-2)\left(1+x+x^{2}\right)(2 F(x, 0)-F(0,0))\right) \\
& \quad=(k-2)\left((1+2 x)(2 F(x, 0)-F(0,0))+2\left(1+x+x^{2}\right) \frac{d}{d x} F(x, 0)\right),
\end{aligned}
$$

and

$$
\begin{aligned}
& \frac{d^{2}}{d x^{2}}\left((k-2)\left(1+x+x^{2}\right)(2 F(x, 0)-F(0,0))\right) \\
& \quad=(k-2)\left(2(2 F(x, 0)-F(0,0))+4(1+2 x) \frac{d}{d x} F(x, 0)+2\left(1+x+x^{2}\right) \frac{d^{2}}{d x^{2}} F(x, 0)\right) .
\end{aligned}
$$

Letting $x=1$ gives

$$
\begin{aligned}
& \left.\frac{d^{2}}{d x^{2}}\left((k-2)\left(1+x+x^{2}\right)(2 F(x, 0)-F(0,0))\right)\right|_{x=1} \\
& \quad=(k-2)\left(2(2 F(1,0)-F(0,0))+12 \frac{d}{d x} F(x, 0)_{x=1}+\left.6 \frac{d^{2}}{d x^{2}} F(x, 0)\right|_{x=1}\right) .
\end{aligned}
$$


By using (15) and (20) the above rewrites

$$
\begin{aligned}
& \left.\frac{d^{2}}{d x^{2}}\left((k-2)\left(1+x+x^{2}\right)(2 F(x, 0)-F(0,0))\right)\right|_{x=1} \\
& \quad=(k-2)\left(\frac{4(k-3)}{3(k-2)}+\frac{2 k}{k-2}+\left.6 \frac{d^{2}}{d x^{2}} F(x, 0)\right|_{x=1}\right) .
\end{aligned}
$$

Equating the r.h.s. of (116) and (117) we obtain

$$
-4+\frac{2 k(k-6)}{k-3}+\left.2(k-3) \frac{d^{2}}{d x^{2}} F(x, x)\right|_{x=1}=(k-2)\left(\frac{4(k-3)}{3(k-2)}+\frac{2 k}{k-2}+\left.6 \frac{d^{2}}{d x^{2}} F(x, 0)\right|_{x=1}\right),
$$

that is

$$
\begin{aligned}
\left.\frac{d^{2}}{d x^{2}} F(x, x)\right|_{x=1} & =\frac{1}{2(k-3)}\left(4-\frac{2 k(k-6)}{k-3}+\frac{4(k-3)}{3}+2 k+\left.6(k-2) \frac{d^{2}}{d x^{2}} F(x, 0)\right|_{x=1}\right), \\
& =\frac{k(2 k+3)}{3(k-3)^{2}}+\left.\frac{3(k-2)}{k-3} \frac{d^{2}}{d x^{2}} F(x, 0)\right|_{x=1},
\end{aligned}
$$

which is (22).

\section{Proof of Lemma 1}

We may rewrite the set $\mathcal{L}$ in $(34)$ as

$$
\mathcal{L}:=\left\{0 \leq \rho \leq 1,-\pi \leq \theta \leq \pi: 2 \rho^{3} \cos \theta-k \rho^{2}+k-2=0\right\} .
$$

Define $f(\theta, \rho):=2 \rho^{3} \cos \theta-k \rho^{2}+k-2$. Let us show that for any $\theta \in \mathbb{R}, f(\theta, \rho)$ has a unique zero in $(0,1]$, denoted by $\rho=\rho(\theta)$.

Assume first that $\theta=2 l \pi$ for $l \in \mathbb{Z}$. From

$$
f(2 l \pi, \rho)=(x-1)\left(x-\frac{k-2-\sqrt{k^{2}+4 k-12}}{2}\right)\left(x-\frac{k-2+\sqrt{k^{2}+4 k-12}}{2}\right),
$$

we deduce that $f(2 l \pi, \rho)$ has a single zero in $(0,1]$ given by $\rho(2 l \pi)=1$.

Assume now that $\theta \in \mathbb{R}-\{2 l \pi, l \in \mathbb{Z}\}$. From

$$
f(\theta,-1)=-2(\cos \theta+1) \leq 0, \quad f(\theta, 0)=k-2>0, \quad f(\theta, 1)=2(\cos \theta-1)<0
$$

and

$\lim _{\rho \rightarrow-\infty} f(\theta, \rho)=\left\{\begin{array}{ll}-\infty, & \text { if } \theta \in(2 l \pi,(2 l+1) \pi), \\ +\infty, & \text { if } \theta \in((2 l+1) \pi, 2(l+1) \pi),\end{array} \quad \lim _{\rho \rightarrow \infty} f(\theta, \rho)= \begin{cases}+\infty, & \text { if } \theta \in(2 l \pi,(2 l+1) \pi), \\ -\infty, & \text { if } \theta \in(2 l+1,2(l+1)),\end{cases}\right.$ 
for $l \in \mathbb{Z}$, we readily deduce that the polynomial $f(\theta, \rho)$ of degree 3 in the variable $\rho$ has a single zero $\rho=\rho(\theta)$ in $(0,1)$. In summary, for any $\theta \in \mathbb{R}, f(\theta, \rho)$ has a unique zero $\rho=\rho(\theta)$ in $(0,1]$.

Since the partial derivatives $\frac{d^{m}}{d \theta^{m}} f(\theta, \rho)$ and $\frac{d^{m}}{d \rho^{m}} f(\theta, \rho)$ exist for all $m=0,1, \ldots$ and $\frac{d}{d \rho} f(\theta, \rho)=$ $2 \rho(3 \rho \cos \theta-k)$ does not vanish for any zero $(\theta, \rho)$ of $f(\theta, \rho)$ in $\mathbb{R} \times(0,1]$ as $k>3$, we conclude from the implicit function theorem $[26]$ that $\rho(\theta)$ is infinitely differentiable in $\mathbb{R}$.

Because $\rho(\theta)=\rho(\theta+2 \pi)$ for any $\theta \in \mathbb{R}$, the function $\rho(\theta)$ is periodic with period $2 \pi$ and is infinitely differentiable in (in particular) $[-\pi, \pi]$. The continuity of $\rho(\theta)$ in $[-\pi, \pi]$ together with $\rho(-\pi)=\rho(\pi)$ implies that the contour $\mathcal{L}$ defined by $(35)$ is closed and the differentiability of $\rho(\theta)$ in $[-\pi, \pi]$ implies that it is smooth. The contour $\mathcal{L}$ is symmetric with respect to the real axis since $f(\theta, \rho)=f(-\theta, \rho)$ for all $\theta \in R, \rho \geq 0$.

Differentiating equation $f(\theta, \rho(\theta))=0$ once gives $\rho^{\prime}(0)=0$ by using $\rho(0)=1$, differentiating it twice gives $\rho^{\prime \prime}(0)=\frac{1}{3-k}$ by using $\rho(0)=1$ and $\rho^{\prime}(0)=0$, and differentiating it three times gives $\rho^{\prime \prime \prime}(0)=0$ by using $\rho(0)=1, \rho^{\prime}(0)=0$ and $\rho^{\prime \prime}(0)=\frac{1}{3-k}$.

Last, if $\mathcal{L}$ intersects with itself this means that there exist $\theta_{1}, \theta_{2} \in(0, \pi), \theta_{1} \neq \theta_{2}$, such that $\rho\left(\theta_{1}\right)=\rho\left(\theta_{2}\right)$ (because $\mathcal{L}$ is symmetric with respect to the real axis we do not need to consider the case where $\left.\theta_{1}, \theta_{2} \in(-\pi, 0)\right)$. Assume that $\rho\left(\theta_{1}\right)=\rho\left(\theta_{2}\right)=\rho_{0}$ for some $\theta_{1}, \theta_{2} \in(0, \pi)$. Note that $\rho_{0} \neq 0$ as $\rho=0$ is not a zero of $f_{\theta}(\rho)$. The identities $f\left(\theta_{i}, \rho_{0}\right)=0$ for $i=1,2$ imply that $2 \rho_{0}^{3}\left(\cos \left(\theta_{1}-\cos \left(\theta_{2}\right)\right)=0\right.$, which in turn implies that $\cos \left(\theta_{1}\right)=\cos \left(\theta_{2}\right)$ and that $\theta_{1}=\theta_{2}$ since $\theta_{1}, \theta_{2} \in(0, \pi)$. Therefore the contour $\mathcal{L}$ does not intersect with itself.

\section{E Proof that $I_{2}=1$}

We have by (55), (38), and (51)

$$
\begin{aligned}
y_{\mathcal{C}} & \frac{\log \left(u^{-1} G(u)\right.}{u} d u=i \int_{-\pi}^{\pi} \log \left(e^{-i \theta} \frac{\rho(\phi(\theta))^{3} e^{i \phi(\theta)}}{1-\rho(\phi(\theta))^{3} e^{-i \phi(\theta)}}\right) d \theta \\
& =i \int_{-\pi}^{0} \log \left(e^{-i \theta} \frac{\rho(\phi(\theta))^{3} e^{i \phi(\theta)}-1}{1-\rho(\phi(\theta))^{3} e^{-i \phi(\theta)}}\right) d \theta+i \int_{-\pi}^{0} \log \left(e^{i \theta} \frac{\rho(\phi(-\theta))^{3} e^{i \phi(-\theta)}-1}{1-\rho(\phi(-\theta))^{3} e^{-i \phi(-\theta)}}\right) d \theta, \\
& =i \int_{-\pi}^{0} \log \left(e^{-i \theta} \frac{\rho(\phi(\theta))^{3} e^{i \phi(\theta)}-1}{1-\rho(\phi(\theta))^{3} e^{-i \phi(\theta)}}\right) d \theta+i \int_{-\pi}^{0} \log \left(e^{i \theta} \frac{\rho(\phi(\theta))^{3} e^{-i \phi(\theta)}-1}{1-\rho(\phi(\theta))^{3} e^{i \phi(\theta)}}\right) d \theta \\
& =i \int_{-\pi}^{0} \log \left(e^{-i \theta} \frac{\rho(\phi(\theta))^{3} e^{i \phi(\theta)}-1}{1-\rho(\phi(\theta))^{3} e^{-i \phi(\theta)}}\right) d \theta-i \int_{-\pi}^{0} \log \left(e^{-i \theta} \frac{\rho(\phi(\theta))^{3} e^{i \phi(\theta)}-1}{1-\rho(\phi(\theta))^{3} e^{-i \phi(\theta)}}\right) d \theta, \\
& =0
\end{aligned}
$$

where we have used (41) and the property that $\rho(\theta)=\rho(-\theta)$ for all $\theta$ (cf. Lemma 1) to establish (118). This concludes the proof from the definition of $I_{2}$ in (55). 


\section{F Properties of mappings $G$ and $g$}

The following results will be used in the proof of Lemmas 3 and 4 . Since $\gamma_{0}$ is a one-to-one mapping from $\mathcal{L}$ onto $\mathcal{C}$, for any $t=e^{i \theta}$ there exists a unique $w \in \mathcal{L}$ such that $\gamma_{0}(w)=t$. Hence, cf. (51)-(52),

$$
\begin{aligned}
G\left(\gamma_{0}(w)\right) & =-\frac{1-\rho(\theta)^{3} e^{i \theta}}{1-\rho(\theta)^{3} e^{-i \theta}} \\
g\left(\gamma_{0}(w)\right) & =\frac{1-\rho(\theta)^{3} \cos \theta}{1-\rho(\theta)^{3} e^{-i \theta}}
\end{aligned}
$$

for all $w \in \mathcal{L}$ by using $\gamma\left(\gamma_{0}(w)\right)=w$ (in particular) for all $w \in \mathcal{L}$.

Introduce the mappings

$$
\begin{aligned}
\tilde{G}(\theta) & :=\frac{\rho(\theta)^{3} e^{i \theta}-1}{1-\rho(\theta)^{3} e^{-i \theta}} \\
\tilde{g}(\theta) & :=\frac{1-\rho(\theta)^{3} \cos \theta}{1-\rho(\theta)^{3} e^{-i \theta}} .
\end{aligned}
$$

Observe from (51), (52) and (38) that for $t=e^{i \theta}$

$$
G(t)=\tilde{G}(\phi(\theta)), \quad g(t)=\tilde{g}(\phi(\theta)) .
$$

\section{Lemma 3 (Properties of $G$ and $g$ )}

The function $G(t)$ does not vanish on $\mathcal{C}$ and $G(t), g(t), \log \left(t^{-1} G(t)\right)$ and $g(t) e^{-H(t)}$ are continuous on $\mathcal{C}$.

Also, $G(1)=1$ and $g(1)=0$.

Proof. Let $t_{0}=e^{i \theta_{0}} \in \mathcal{C}$. Assume first that $\theta_{0} \in[-\pi, \pi]-\{0\}$. Since $\phi(\theta)$ is continuous in $[-\pi, \pi]$, the functions $\tilde{G}(\phi(\theta))$ and $\tilde{g}(\phi(\theta))$ in (121)-(122) are continuous at $\theta_{0}$ if their common denominator $1-\rho(\phi(\theta))^{3} e^{-i \phi(\theta)}$ does not vanish at this point. This is true as $\rho(\theta)<1$ for all $\theta \in[-\pi, \pi]-\{0\}$ and that $\phi(\theta)=0$ iff $\theta=0$. The same argument shows that the numerator of $\tilde{G}(\phi(\theta))$ does not vanish at $\theta_{0}$. Assume now that $\theta_{0}=0$. Applications of L'Hôpital's rule show that $\tilde{G}(0)=1$ (or equivalently $G(1)=1$ ) and $\tilde{g}(0)=1$ (or equivalently $g(1)=0$ ), thereby proving all statements of the lemma (but the continuity of $\left.g(t) e^{-H(t)}\right)$ thanks to $(123)$.

It remains to show that $g(t) e^{-H(t)}$ with $H(t)$ defined in $(60)$ is continuous on $\mathcal{C}$. We have just shown that the first term $\log \left(t^{-1} G(t)\right)$ in (60) is continuous on $\mathcal{C}$. Rewriting the integral in (60) as $\int_{-\pi}^{\pi} \frac{\log \left(e^{-i \theta} G\left(e^{i \theta}\right)\right)-\log \left(t^{-1} G(t)\right)}{e^{i \theta}-t} e^{-i \theta} d \theta$, we observe that it is a continuous function of $t \in \mathcal{C}$ since for each

$\theta \in[-\pi, \pi]$ the mapping $t \rightarrow \frac{\log \left(e^{-i \theta} G\left(e^{i \theta}\right)\right)-\log \left(t^{-1} G(t)\right)}{e^{i \theta}-t} e^{-i \theta}$ is continuous on $\mathcal{C}$ and the range of integration (i.e., $[-\pi, \pi])$ is finite. Therefore $H(t)$ is continuous on $\mathcal{C}$ as the sum of two continuous functions on $\mathcal{C}$, and so is $g(t) e^{-H(t)}$ as the composition of continuous functions on $\mathcal{C}$. This ends the proof. 


\section{G Calculation of the index $\chi$}

\section{Lemma 4 (Index)}

The index $\chi$ (defined in (53)) is equal to one.

Proof. Thanks to (119) the index $\chi$ in (53) is also given by

$$
\chi=\frac{1}{2 \pi} \arg \left[G\left(\gamma_{0}(w)\right)\right]_{\mathcal{L}} .
$$

Take $w=\rho(\theta) e^{i \theta} \in \mathcal{L}$. When $\theta=\pi$ (resp. $\left.\theta=-\pi\right), w=-\rho(\pi)(w=\rho(-\pi)=\rho(\pi))$ and $G\left(\gamma_{0}(w)\right)=$ $-\frac{1+\rho(\pi)^{3}}{1+\rho(\pi)^{3}}=-1$. When $\theta=0, w=\rho(0)=1$ and

$$
G\left(\gamma_{0}(1)\right)=-\lim _{\theta \rightarrow 0} \frac{-3 \frac{d}{d \theta} \rho(\theta) \rho(\theta)^{2} e^{i \theta}-i \rho(\theta)^{3} e^{i \theta}}{-3 \frac{d}{d \theta} \rho(\theta) \rho(\theta)^{2} e^{-i \theta}+i \rho(\theta)^{3} e^{-i \theta}}=1,
$$

since $\left.\frac{d}{d \theta} \rho(\theta)\right|_{\theta=0}=0$ and $\rho(\theta)=1$ by (36), where the second equality holds by L'Hôpital's rule.

The function $G\left(\gamma_{0}(w)\right)=-\frac{1-\rho(\theta)^{3} e^{i \theta}}{1-\rho(\theta)^{3} e^{-i \theta}}$ in $(119)$ is continuous in $[-\pi, \pi]$ since $\rho(\theta)$ is continuous in $[-\pi, \pi]$ (cf. Lemma 1), since its denominator does not vanish for $\theta \neq 0$ as $\rho(\theta)<1$ for $\theta \neq 0$ and since $\lim _{\theta \rightarrow 0}-\frac{1-\rho(\theta)^{3} e^{i \theta}}{1-\rho(\theta)^{3} e^{-i \theta}}=1$ as shown above.

Let us now show that $G\left(\gamma_{0}(w)\right)$ crosses the real axis only at $\theta=0$ and at $\theta=\pi$ (resp. $\left.\theta=-\pi\right)$ when $w$ describes once the contour $\mathcal{L}$ or, equivalently, that $\Im\left(G\left(\gamma_{0}(w)\right) \neq 0\right.$ when $\theta \in(-\pi, \pi)$ with $\theta \neq 0$. By (119)

$$
\Im\left(G\left(\gamma_{0}(w)\right)=\frac{2\left(1-\rho(\theta)^{3} \cos \theta\right) \rho(\theta)^{3} \sin \theta}{1-2 \rho(\theta)^{3} \cos \theta+\rho(\theta)^{6}} .\right.
$$

Fix $\theta_{0} \in(-\pi, \pi)$ with $\theta_{0} \neq 0$. The numerator of $\Im\left(G\left(\gamma_{0}(w)\right)\right.$ vanishes if and only if $1=\rho\left(\theta_{0}\right)^{3} \cos \left(\theta_{0}\right)$. Since $\rho(\theta)<1$ for $\theta \neq 0$, we see that $1>\rho\left(\theta_{0}\right)^{3} \cos \left(\theta_{0}\right)$. On the other hand, the denominator of $\Im\left(G\left(\gamma_{0}(w)\right)\right.$ does not vanish since $1-2 \rho\left(\theta_{0}\right)^{3} \cos \left(\theta_{0}\right)+\rho\left(\theta_{0}\right)^{6}>1-2 \rho\left(\theta_{0}\right)^{3}+\rho\left(\theta_{0}\right)^{6}=\left(1-\rho\left(\theta_{0}\right)^{3}\right)^{2}>0$ by using $\cos \left(\theta_{0}\right)<1$ and $\rho\left(\theta_{0}\right)<1$. Therefore, $\Im\left(G\left(\gamma_{0}(w)\right)>0\right.$ for $\theta_{0} \in(0, \pi)$ and $\Im\left(G\left(\gamma_{0}(w)\right)<0\right.$ for $\theta_{0} \in(-\pi, 0)$.

In summary, we have shown that as $w$ describes once the contour $\mathcal{L}$ the mapping $G\left(\gamma_{0}(w)\right)$ describes once a circuit around zero in the counter-clockwise direction, thereby proving that $\chi=1$ by (124).

\section{H Hölder condition for first and second derivatives of $\alpha$ and $\beta$}

Lemma 5 The mapping $\phi$ defined in (39) is twice differentiable in $[-\pi, \pi]$ and its second derivative is continuous in $[-\pi, \pi]$.

Proof. We recall that the stability condition $k \geq 4$ is enforced. By Lemma 3 in [7, p. 876], the conformal mapping $\gamma(z)$ from $\mathcal{C}$ onto $\mathcal{L}$ (see Section 5.1) is differentiable on $\mathcal{C}$. By (98) and the differentiability of 
$\gamma$ on $\mathcal{C}, \phi^{\prime}(\theta)$ exists if (a) $\rho^{\prime}(\phi(\theta))+i \rho(\phi(\theta)) \neq 0$ for $\theta \in[-\pi, \pi]$. The latter is always true since $\rho^{\prime}(\theta)$ is a real number (cf. (96)) and $\rho(\theta) \neq 0$ for all $\theta \in[-\pi, \pi]$. This proves the differentiability of $\phi$ on $[-\pi, \pi]$.

The proof that $\phi$ is twice differentiable is more tedious. Differentiating the equation $\gamma\left(\gamma_{0}(w)\right)=w$ for $w \in \mathcal{L}$ (see Section 5.1) gives $\gamma_{0}^{\prime}(w)=\frac{1}{\gamma^{\prime}\left(\gamma_{0}(w)\right)}$. But Lemma 3 in [7, p. 876] also tells us that $\gamma^{\prime}(z) \neq 0$ on $\mathcal{C}-\{1\}$ for all $k \geq 4$, which allows to conclude that

$$
\gamma_{0}^{\prime}(w) \neq 0, \quad \forall w \in \mathcal{L}-\{1\}
$$

By (127) the denominator in (99) does not vanish for $\theta \in[-\pi, \pi]-\{0\}$ and it does not vanish either for $\theta=0$ since $\gamma_{0}^{\prime}\left(\rho(\phi(0)) e^{i \phi(0)}\right)=\gamma_{0}^{\prime}(1) \neq 0$ by (153) (here we are using that $\rho^{\prime}(\phi(\theta))+i \rho(\phi(\theta)) \neq 0$ for $\theta \in[-\pi, \pi]$ as already observed). On the other hand, a glance at the r.s.h. of (99) indicates that the term within square brackets is well-defined on $[-\pi, \pi]$ if $\left(\right.$ c) $\gamma_{0}^{\prime \prime}(w)$ is well-defined on $\mathcal{L}$ which, by (148) with $i=2$, (150) and (152), amounts to show that (c) $K-w \delta^{2} \neq 0$ for $w \in \mathcal{L}$ in the denominator of (152) (Hint: $w \notin \mathcal{L}$ and $K-w$ does not vanish on $\mathcal{L}$ since $K=k-2 \geq 2$ and $|w| \leq 1$ - see Figure 3 ). It can be checked that property (c) holds (a more direct argument is to notice that (c) holds as otherwise $\gamma_{0}$ in $(44)$ would not be well-defined on $\mathcal{L})$. This proves that $\phi$ is twice differentiable on $\theta \in[-\pi, \pi]$. Last, we observe from $(99)$ that $\phi^{\prime \prime}(\theta)$ is continuous on $[-\pi, \pi]$ as it is only composed by continuous mappings, which completes the proof.

Lemma 6 The mapping $\alpha$ defined in (82) is twice differentiable on $\mathcal{C}$ and its second derivative is continuous on $\mathcal{C}$. The same result holds for the mapping $\beta$ defined in (83).

Proof. Define

$$
\begin{aligned}
\tilde{G}(\theta) & =-\frac{1-\rho(\theta)^{3} e^{i \theta}}{1-\rho(\theta)^{3} e^{-i \theta}}, \\
\tilde{g}(\theta) & =\frac{1-\rho(\theta)^{3} \cos \theta}{1-\rho(\theta)^{3} e^{-i \theta}} .
\end{aligned}
$$

Since $\rho(\theta) \in[0,1)$ for $\theta \in(0,1)$ and $\rho(0)=1$ (see Section 5.1), the denominator in the r.h.s. of (128) and (129) has a single zero of multiplicity one in $[-\pi, \pi]$ at $\theta=0$. Since both numerators in the r.h.s. of (128) and (129) vanish at $\theta=0$, this shows that the mappings $\tilde{G}(\theta)$ and $\tilde{g}(\theta)$ are continuous in $[-\pi, \pi]$.

Notice that (cf. (51), (52), (38))

$$
G\left(e^{i \theta}\right)=\tilde{G}(\phi(\theta)) \quad \text { and } \quad g\left(e^{i \theta}\right)=\tilde{g}(\phi(\theta)) .
$$

We have for $u=e^{i \theta}$ (cf. (82))

$$
\alpha^{\prime}(u)=\frac{d}{d e^{i \theta}} \log \left(e^{-i \theta} G\left(e^{i \theta}\right)\right)=-i e^{-i \theta} \frac{d}{d \theta} \log \left(e^{-i \theta} \tilde{G}(\phi(\theta))=-i e^{-i \theta}\left(-i+\frac{\phi^{\prime}(\theta) \tilde{G}^{\prime}(\phi(\theta))}{\tilde{G}(\phi(\theta))}\right)\right.
$$


and

$$
\begin{aligned}
\alpha^{\prime \prime}(u) & =\frac{d}{d u} \alpha^{\prime}(u)=\frac{1}{i e^{i \theta}} \frac{d}{d \theta} \alpha^{\prime}(u)=-i e^{-i \theta} \frac{d}{d \theta}\left\{-i e^{-i \theta}\left(-i+\frac{\phi^{\prime}(\theta) \tilde{G}^{\prime}(\phi(\theta))}{\tilde{G}(\phi(\theta))}\right)\right\}, \quad \text { by using }(130), \\
& =-i e^{-i \theta}\left(-e^{-i \theta}\left(-i+\frac{\phi^{\prime}(\theta) \tilde{G}^{\prime}(\phi(\theta))}{\tilde{G}(\phi(\theta))}\right)-i e^{-i \theta} \frac{d}{d \theta} \frac{\phi^{\prime}(\theta) \tilde{G}^{\prime}(\phi(\theta))}{\tilde{G}(\phi(\theta))}\right), \\
& =-e^{-i \theta} \alpha^{\prime}(u)-e^{-2 i \theta} \frac{d}{d \theta} \frac{\phi^{\prime}(\theta) \tilde{G}^{\prime}(\phi(\theta))}{\tilde{G}(\phi(\theta))}, \quad \text { by using again }(130), \\
& =-e^{-i \theta} \alpha^{\prime}(u)-e^{-2 i \theta}\left(\phi^{\prime \prime}(\theta) \frac{\tilde{G}^{\prime}(\phi(\theta))}{\tilde{G}(\phi(\theta))}+\phi^{\prime}(\theta)^{2} \frac{\tilde{G}^{\prime \prime}(\phi(\theta))}{\tilde{G}(\phi(\theta))}-\phi^{\prime}(\theta)^{2}\left(\frac{\tilde{G}^{\prime}(\phi(\theta))}{\tilde{G}(\phi(\theta))}\right)^{2}\right)
\end{aligned}
$$

after easy algebra. Similarly for $u=e^{i \theta}$ (cf. (83))

$$
\begin{aligned}
\beta^{\prime}(u) & =\frac{d}{d e^{i \theta}}\left\{g\left(e^{i \theta}\right) e^{-H\left(e^{i \theta}\right)}\right\}=-i e^{-i \theta} \frac{d}{d \theta}\left\{\tilde{g}(\phi(\theta)) e^{-H\left(e^{i \theta}\right)}\right\} \\
& =-i e^{-i \theta}\left(\phi^{\prime}(\theta) \tilde{g}^{\prime}(\phi(\theta))-i e^{i \theta} \tilde{g}(\phi(\theta)) H^{\prime}\left(e^{i \theta}\right) e^{-H\left(e^{i \theta}\right)}\right.
\end{aligned}
$$

and

$$
\begin{aligned}
\beta^{\prime \prime}(u)= & -e^{-2 i \theta}\left[-i \phi^{\prime}(\theta) \tilde{g}^{\prime}(\phi(\theta))+\phi^{\prime \prime}(\theta) \tilde{g}^{\prime}(\phi(\theta))+\phi^{\prime}(\theta)^{2} \tilde{g}^{\prime \prime}(\phi(\theta))-i e^{i \theta} \phi^{\prime}(\theta) \tilde{g}^{\prime}(\phi(\theta)) H^{\prime}\left(e^{i \theta}\right)\right. \\
& \left.+e^{2 i \theta} \tilde{g}(\phi(\theta)) H^{\prime \prime}\left(e^{i \theta}\right)-e^{i \theta}\left(i \phi^{\prime}(\theta) \tilde{g}^{\prime}(\phi(\theta))+e^{i \theta} \tilde{g}(\phi(\theta)) H^{\prime}\left(e^{i \theta}\right)\right) H^{\prime}\left(e^{i \theta}\right)\right] e^{-H\left(e^{i \theta}\right)},
\end{aligned}
$$

after lengthy but easy algebra. On the other hand,

$$
\begin{aligned}
\tilde{G}^{\prime}(\theta) & =-\frac{2 i \rho(\theta)^{2}}{\left(1-\rho(\theta)^{3} e^{-i \theta}\right)^{2}}\left(\rho(\theta)^{4}-\rho(\theta) \cos \theta-3 \rho^{\prime}(\theta) \sin \theta\right), \\
\tilde{g}^{\prime}(\theta) & =\frac{i \rho(\theta)^{2}}{\left(1-\rho(\theta)^{3} e^{-i \theta}\right)^{2}}\left(\rho(\theta)^{4}-\rho(\theta) \cos \theta-3 \rho^{\prime}(\theta) \sin \theta\right),
\end{aligned}
$$

so that

$$
\tilde{G}^{\prime}(\theta)=-2 \tilde{g}^{\prime}(\theta)
$$


and

$$
\begin{aligned}
\tilde{G}^{\prime \prime}(\theta)= & \frac{\rho(\theta)}{\left(1-\rho(\theta)^{3} e^{-i \theta}\right)^{3}}\left(-4 \rho(\theta)^{8} e^{-i \theta}+\rho(\theta)^{5}\left(3+e^{-2 i \theta}\right)\right. \\
& +3 \rho(\theta)^{4}\left(2 i \rho^{\prime}(\theta)\left(e^{-2 i \theta}-3\right)-\rho^{\prime \prime}(\theta)\left(1-e^{-2 i \theta}\right)\right)+12 \rho(\theta)^{3} \rho^{\prime}(\theta)^{2}\left(1-e^{-2 i \theta}\right) \\
& \left.-2 i \rho(\theta)^{2} \sin \theta+6 i \rho(\theta)\left(2 \rho^{\prime}(\theta) \cos \theta+\rho^{\prime \prime}(\theta) \sin \theta\right)+12 i \rho^{\prime}(\theta)^{2} \sin \theta\right), \\
\tilde{g}^{\prime \prime}(\theta)= & -\frac{1}{2} \tilde{G}^{\prime \prime}(\theta) \quad \text { from }(136) .
\end{aligned}
$$

In particular,

$$
\tilde{G}^{\prime}(0)=i \frac{k}{k-3}, \quad \tilde{G}^{\prime \prime}(0)=-\left(\frac{k}{k-3}\right)^{2}, \quad \tilde{g}^{\prime}(0)=-\frac{i}{2} \cdot \frac{k}{k-3}, \quad \tilde{g}^{\prime \prime}(0)=\frac{1}{2}\left(\frac{k}{k-3}\right)^{2} .
$$

We are now in position to prove the lemma. We have shown in Lemma 5 above that $\phi(\theta)$ is twice differentiable in $[-\pi, \pi]$ and that its second derivative is continuous in $[-\pi, \pi]$. Also, recall that $\rho(\theta)$ is infinitely differentiable in $[-\pi, \pi]$ (cf. Lemma 1 ).

We start with $\alpha$. The first derivative of $\alpha(u)$ in (130) is continuous on $\mathcal{C}$ if the numerator in the ratio ${ }^{4}$

$$
\frac{\tilde{G}^{\prime}(\theta)}{\tilde{G}(\theta)}=\frac{2 i \rho(\theta)^{2}}{\left(1-\rho(\theta)^{3} e^{-i \theta}\right)\left(1-\rho(\theta)^{3} e^{i \theta}\right)}\left(\rho(\theta)^{4}-\rho(\theta) \cos \theta-3 \rho^{\prime}(\theta) \sin \theta\right),
$$

vanishes when the denominator vanishes. Since $\rho(\theta) \in[0,1)$ for $\theta \neq 0$ and $\rho(0)=1$ (see Lemma 1 ) the denominator in (139) has a single zero in $[-\pi, \pi]$ at $\theta=0$ and this zero has multiplicity two. It is easily checked that $\rho(\theta)^{4}-\rho(\theta) \cos \theta-3 \rho^{\prime}(\theta) \sin \theta$ has a zero of order two at $\theta=0$. This shows that $\alpha^{\prime}$ is well-defined (actually continuous) on $\mathcal{C}$.

Now, since $\alpha^{\prime}$ is continuous on $\mathcal{C}$, we see from (131) and from the fact that the ratio in (134) is continuous for $\theta \in[-\pi, \pi]$, that $\alpha^{\prime \prime}$ is continuous on $\mathcal{C}$ if the numerator in the ratio

$$
\begin{aligned}
\frac{\tilde{G}^{\prime \prime}(\theta)}{\tilde{G}(\theta)} & =-\frac{\rho(\theta)}{\left(1-\rho(\theta)^{3} e^{-i \theta}\right)^{2}\left(1-\rho(\theta)^{3} e^{i \theta}\right)} \\
& \times\left(-4 \rho(\theta)^{8} e^{-i \theta}+\rho(\theta)^{5}\left(3+e^{-2 i \theta}\right)+3 \rho(\theta)^{4}\left(2 i \rho^{\prime}(\theta)\left(e^{-2 i \theta}-3\right)-\rho^{\prime \prime}(\theta)\left(1-e^{-2 i \theta}\right)\right)\right. \\
& \left.+12 \rho(\theta)^{3} \rho^{\prime}(\theta)^{2}\left(1-e^{-2 i \theta}\right)-2 i \rho(\theta)^{2} \sin \theta+6 i \rho(\theta)\left(2 \rho^{\prime}(\theta) \cos \theta+\rho^{\prime \prime}(\theta) \sin \theta\right)+12 i \rho^{\prime}(\theta)^{2} \sin \theta\right)
\end{aligned}
$$

\footnotetext{
${ }^{4}$ Since the mapping $\phi(\theta)$ is strictly increasing in $[-\pi, \pi]$ with $\phi(-\pi)=-\pi$ and $\phi(\pi)=\pi$ (see Section 5.1) showing that the mapping $\varphi(\theta)$ is continuous in $[-\pi, \pi]$ is the same, thanks to the continuity of $\phi(\theta)$, as showing that $\varphi(\phi(\theta))$ is continuous in $[-\pi, \pi]$. This property is used repeatedly hereafter.
} 
vanishes when the denominator vanishes. As observed above the denominator in (140) has a single zero in $[-\pi, \pi]$ at $\theta=0$ and this zero has multiplicity three. One can check that the term within the parentheses in (140) has a zero of order three at $\theta=0$ (Hint: $\rho^{\prime}(0)=\rho^{\prime \prime \prime}(0)=0$ and $\rho^{\prime \prime}(0)=1 /(3-k)$ from Lemma 1 ), which proves that $\alpha$ is twice differentiable on $\mathcal{C}$ and that $\alpha^{\prime \prime}$ is continuous on $\mathcal{C}$.

We now turn our attention to $\beta$. It is easy to show that $\tilde{g}$ is twice differentiable in $[-\pi, \pi]$ and that its second derivative is continuous (Hint: check that the numerator in (135) (resp. (138)) vanishes at $\theta=0$ as many times as the denominator does). We are therefore left with proving that the mapping $H$ is twice differentiable on $\mathcal{C}$ and that its second derivative is continuous on $\mathcal{C}$. With $(82), H(t)$ in $(60)$ writes

$$
H(t)=\frac{1}{2} \alpha(t)+\frac{1}{2 \pi i} \int_{\mathcal{C}} \frac{\alpha(u)}{u-t} d u .
$$

Thanks to $(74), H(t)$ in (141) is also given by

$$
H(t)=\alpha(t)+\frac{1}{2 \pi i} \int_{\mathcal{C}} \frac{\alpha(u)-\alpha(t)}{u-t} d u, \quad t \in \mathcal{C} .
$$

For every $t \in \mathcal{C}$, notice that the integrand in (142) is continuous on $\mathcal{C}$. This is clearly true for $u \neq t$. When $u=t$ it is equal to $\alpha^{\prime}(t)$ by L'Hôpital's rule. Differentiating (142) gives, for $t \in \mathcal{C}$,

$$
\begin{aligned}
H^{\prime}(t) & =\alpha^{\prime}(t)+\frac{1}{2 \pi i} \int_{\mathcal{C}} \frac{\alpha(u)-\alpha(t)-\alpha^{\prime}(t)(u-t)}{(u-t)^{2}} d u, \\
H^{\prime \prime}(t) & =\alpha^{\prime \prime}(t)+\frac{1}{2 \pi i} \int_{\mathcal{C}} \frac{2(\alpha(u)-\alpha(t))-2 \alpha^{\prime}(t)(u-t)-\alpha^{\prime \prime}(t)(u-t)^{2}}{(u-t)^{3}} d u .
\end{aligned}
$$

For every $t \in \mathcal{C}$, notice that the integrand in (143) (resp. (144)) is continuous on $\mathcal{C}$. This is clearly true for $u \neq t$. When $u=t$ it is equal to $\frac{1}{2} \alpha^{\prime \prime}(t)$ (resp. $\frac{1}{3} \alpha^{\prime \prime \prime}(t)$ ) by L'Hôpital's rule. The third derivative $\alpha^{\prime \prime \prime}(t)$ can be calculated similarly to the calculation of $\alpha^{\prime \prime}$ in (131); this calculation is omitted (for the sake of simplicity we recommend discarding the point $u=t$ when evaluating the integral in (144)). Since we have shown that $\alpha, \alpha^{\prime}$ and $\alpha^{\prime \prime}$ are all continuous on $\mathcal{C}$, the mappings $H, H^{\prime}$ and $H^{\prime \prime}$ are all continuous on $\mathcal{C}$ as the range of integration in (142)-(144) is bounded. This concludes the proof.

\section{First and second derivatives of $\gamma_{0}$}

Set $K=k-2$. First rewrite $\gamma_{0}(w)$ in $(44)$ as

$$
\gamma_{0}(w)=1-\frac{2}{(1-\delta)^{2}(K-\delta)}\left(\delta \gamma_{0,1}(w)+\gamma_{0,2}(w)\right)
$$

with

$$
\begin{aligned}
\gamma_{0,1}(w) & =\frac{(1-w)^{2}(K-w)}{w} \\
\gamma_{0,2}(w) & =\frac{(w-\delta)(1-w)}{w} \sqrt{\left(K-w \delta^{2}\right)(K-w)}
\end{aligned}
$$


For $i=1,2$ we find

$$
\frac{d^{i}}{d w^{i}} \gamma_{0}(w)=-\frac{2}{(1-\delta)^{2}(K-\delta)}\left(\delta \frac{d^{i}}{d w^{i}} \gamma_{0,1}(w)+\frac{d^{i}}{d w^{i}} \gamma_{0,2}(w)\right)
$$

with

$$
\begin{aligned}
\frac{d}{d w} \gamma_{0,1}(w) & =\frac{(w-1)\left(K w-2 w^{2}+K\right)}{w^{2}} \\
\frac{d^{2}}{d w^{2}} \gamma_{0,1}(w) & =\frac{2\left(K-w^{3}\right)}{w^{3}}
\end{aligned}
$$

and

$$
\begin{aligned}
\frac{d}{d w} \gamma_{0,2}(w)= & \frac{1}{2 w^{2} \sqrt{\left(K-w \delta^{2}\right)(K-w)}}\left(-K \delta^{3} w^{2}+3 K \delta^{2} w^{3}+2 \delta^{3} w^{3}-4 \delta^{2} w^{4}-K \delta^{3} w-K \delta^{2} w^{2}\right. \\
& \left.+2 \delta^{2} w^{3}-2 K^{2} w^{2}-K \delta w^{2}+3 K w^{3}+2 K^{2} \delta-K \delta w-K w^{2}\right) \\
\frac{d^{2}}{d w^{2}} \gamma_{0,2}(w)= & -\frac{1}{4 w^{3}\left(\left(K-w \delta^{2}\right)(K-w)\right)^{3 / 2}}\left(K^{2} \delta^{5} w^{3}+3 K^{2} \delta^{4} w^{4}-12 K \delta^{4} w^{5}+8 \delta^{4} w^{6}+3 K^{2} \delta^{5} w^{2}\right. \\
& +K^{2} \delta^{4} w^{3}-4 K \delta^{5} w^{3}-4 K^{3} \delta^{2} w^{3}-2 K^{2} \delta^{3} w^{3}+18 K^{2} \delta^{2} w^{4}-12 K \delta^{2} w^{5}-12 K^{3} \delta^{3} w \\
& +18 K^{2} \delta^{3} w^{2}-2 K^{2} \delta^{2} w^{3}-4 K \delta^{3} w^{3}-4 K^{3} w^{3}+K^{2} \delta w^{3}+3 K^{2} w^{4}+8 K^{4} \delta \\
& \left.-12 K^{3} \delta w+3 K^{2} \delta w^{2}+K^{2} w^{3}\right)
\end{aligned}
$$

It is easy to see that

$$
\left.\frac{d}{d w} \gamma_{0}(w)\right|_{w=1}=\frac{8 \sqrt{2(k-3)}}{\sqrt{6-k+\sqrt{(k+6)(k-2)}} \cdot(3 \sqrt{k-2}+\sqrt{k+6}))} .
$$

\section{References}

[1] S. Armstrong, J-F. Morizur, J. Janousek, B. Hage, N. Treps, P. K. Lam, and H-A. Bachor. Programmable Multimode Quantum Networks. Nature Communications, 3:1026, 2012.

[2] K. Avrachenkov, P. Nain, and U. Yechiali. A retrial system with two input streams and two orbit queues. Queueing Systems (QUESTA), 77(1):1-31, 2014.

[3] C. H. Bennett and G. Brassard. Quantum Cryptography: Public Key Distribution and Coin Tossing. Theoretical Computer Science, 560(P1):7-11, 2014. 
[4] C. H. Bennett, G. Brassard, and N. D. Mermin. Quantum cryptography without Bell's theorem. Physical Review Letters, 68(5):557, 1992.

[5] M. K. Bhaskar, R. Riedinger, B. Machielse, D. S. Levonian, C. T. Nguyen, E. N. Knall, H. Park, D. Englund, M. Lončar, D. D. Sukachev, and M. D. Lukin. Experimental demonstration of memoryenhanced quantum communication. Nature, 580(7801):60-64, Mar 2020. doi: 10.1038/s41586-0202103-5.

[6] J. P. C. Blanc. Application of the Theory of Boundary Value Problems in the Analysis of a Queueing Model with Paired Services. In Mathematical Centre Tract, volume 153. Mathematish Centrum, Amsterdam, 1982.

[7] J. P. C. Blanc. Asymptotic analysis of a queueing system with a two-dimensional state space. Journal of Applied Probability, 21(4):870-886, 1984.

[8] J. P. C. Blanc, R. Iasnogorodski, and P. Nain. Analysis of the M/GI/1 $\rightarrow$./M/1 queueing model. Queueing Systems (QUESTA), 3:129-156, 1988.

[9] A. Broadbent, J. Fitzsimons, and E. Kashefi. Universal blind quantum computation. In 2009 50th Annual IEEE Symposium on Foundations of Computer Science, pages 517-526. IEEE, 2009.

[10] J. W. Cohen and O. J. Boxma. The M/G/1 queue with alternating service formulated as a RiemannHilbert problem. In F. J. Kylstra, editor, Proc. Performance' 81,, pages 181-199. North Holland Publ. Co., Amsterdam, 1981.

[11] W. J. Cohen and O. J. Boxma. Boundary Value Problems in Queueing System Analysis. North Holland, Amsterdam, 1983.

[12] A. K. Ekert. Quantum Cryptography Based on Bell's Theorem. Physical Review Letters, 67(6):661, 1991.

[13] G. Fayolle, I. Iasnogorodski, and I. Mitrani. The Distribution of Sojourn Times in a Queueing N etwork with Overtaking: Reduction to a Boundary Value Problem. In A. K. Agrawala and S. K. Tripathi, editors, Proc. Performance'83, pages 477-486, College Park, MD, USA, May 25-27 1983. North Holland Pub.

[14] G. Fayolle, R. Iasnogorodski, and V. A. Malyshev. Random Walks in the Quarter Plane. Algebraic Methods, Boundary Value Problems and Applications. In Probability Theory and Stochastic Modeling series, volume 40. Springer, 2nd Edition, 2017.

[15] G. Fayolle and R. Iasnogoroski. Two coupled processors: The reduction to a Riemann-Hilbert problem. Z. Wahrscheinlichkeitstheorie verw. Gebiete, 47:325-351, 1979.

[16] D. Gaier. Konstruktive Methoden der konformen Abbildung. Springer Verlag, Berlin, 1964.

[17] F. D. Gakhov. Boundary Value Problems. Pergamon Press, 1966. 
[18] V. Giovannetti, S. Lloyd, and L. Maccone. Advances in Quantum Metrology. Nature Photonics, $5(4): 222,2011$.

[19] M. A Hall, J. B. Altepeter, and P. Kumar. Ultrafast Switching of Photonic Entanglement. Physical Review Letters, 106(5):053901, 2011.

[20] I. Herbauts, B. Blauensteiner, A. Poppe, T. Jennewein, and H. Huebel. Demonstration of Active Routing of Entanglement in a Multi-User Network. Optics Express, 21(23):29013-29024, 2013.

[21] W. J. Hopp and J. T. Simon. Bounds and heuristics for assembly-like queues. Queueing Systems (QUESTA), 4(2):137-155, 1989.

[22] L. Jiang, J. M. Taylor, A. S. Sørensen, and M. D. Lukin. Distributed quantum computation based on small quantum registers. Physical Review A, 76(6):062323, 2007.

[23] Y. Lee, E. Bersin, A. Dahlberg, S. Wehner, and D. Englund. A Quantum Router Architecture for High-Fidelity Entanglement Flows in Multi-User Quantum Networks. arXiv preprint arXiv:2005.01852, 2020.

[24] D. Leibfried, Murray D. Barrett, T. Schaetz, J. Britton, J. Chiaverini, W. M. Itano, J. D. Jost, C. Langer, and D. J. Wineland. Toward Heisenberg-Limited Spectroscopy with Multiparticle Entangled States. Science, 304(5676):1476-1478, 2004.

[25] R. Li, L. Petit, David P. Franke, J. P. Dehollain, J. Helsen, M. Steudtner, N. K. Thomas, Z. R. Yoscovits, K. J. Singh, S. Wehner, et al. A Crossbar Network for Silicon Quantum Dot Qubits. Science Advances, 4(7):eaar3960, 2018.

[26] A. I. Markushevich. Theory of Functions of a Complex Variable, volume 3, 2nd Edition. Chelsea, New York, 1977.

[27] N. I. Muskhelichvili. Singular Integral Equations. Noordhoff, Groningen, Holland (English translation), 1946.

[28] P. Nain, G. Vardoyan, S. Guha, and D. Towsley. On the Analysis of a Multipartite Entanglement Distribution Switch. Proc. ACM Sigmetrics 2020, in Proceedings of the ACM on Measurement and Analysis of Computing Systems (POMACS), 4(2), June 2020 (Article 23).

[29] M. A. Nielsen and I. Chuang. Quantum Computation and Quantum Information. Cambridge University Press, 2010.

[30] M. Pant, H. Krovi, D. Towsley, L. Tassiulas, L. Jiang, P. Basu, D. Englund, and S. Guha. Routing Entanglement in the Quantum Internet. Quantum Information, 5(Article 25), 2019.

[31] S. Ramachandran and D. Delen. Performance analysis of a kitting process in stochastic assembly systems. Computers $\& 3$ Operations Research, 32(3):449-463, 2005. 
[32] F. Rozpedek, K. Goodenough, J. Ribeiro, N. Kalb, V. Caprara Vivoli, A. Reiserer, R. Hanson, S. Wehner, and D. Elkouss. Parameter regimes for a single sequential quantum repeater. Quantum Science and Technology, 3, 034002, 2018.

[33] E. Schoute, L. Mancinska, T. Islam, I. Kerenidis, and S. Wehner. Shortcuts to Quantum Network Routing. Technical report, Cornell University, October 2016.

[34] P. Som, W. E. Wilhelm, and R. L. Disney. Kitting process in a stochastic assembly system. Queueing Systems (QUESTA), 17(3-4):471-490, 1994.

[35] R. Van Meter. Quantum Networking. John Wiley \& Sons, 2014.

[36] G. Vardoyan, S. Guha, P. Nain, and D. Towsley. On the Exact Analysis of an Idealized Quantum Switch. Performance Evaluation, 144, December 2020 (Article 102141).

[37] G. Vardoyan, S. Guha, P. Nain, and D. Towsley. On the Stochastic Analysis of a Quantum Entanglement Distribution Switch. IEEE Transactions on Quantum Engineering, 2021. 